\title{
Pacific
}

Journal of

Mathematics

\section{ORBIT CORRESPONDENCES FOR}

REAL REDUCTIVE DUAL PAIRS

\author{
SHU-YEN PAN
}




\title{
ORBIT CORRESPONDENCES FOR REAL REDUCTIVE DUAL PAIRS
}

\author{
SHU-YEN PAN
}

\begin{abstract}
Suppose that one of the real vector spaces $V$ and $W$ is symplectic and the other is quadratic. Let $\mathfrak{g}_{1}$ and $\mathfrak{g}_{2}$ denote the Lie algebras of the groups of isometries of the two spaces, and let $\tau_{i}: V \otimes_{\mathbb{R}} W \rightarrow \mathfrak{g}_{i}$ be their respective moment maps for $i=1,2$. Suppose that $\sigma$ and 2 are nilpotent orbits in $\mathfrak{g}_{1}$ and $\mathfrak{g}_{2}$, respectively. We prove that $\tau_{2}\left(\tau_{1}^{-1}(\overline{\mathcal{O}})\right)$ and $\left.\tau_{1}\left(\tau_{2}^{-1}(\overline{2})\right)\right)$ are each the union of at most two closures of nilpotent orbits in $\mathfrak{g}_{1}$ and $\mathfrak{g}_{2}$, respectively (where $\overline{\mathscr{P}}$ denotes the closure of a nilpotent orbit $\mathscr{P}$ ).
\end{abstract}

\section{Introduction}

Let $F$ denote either complex numbers $\mathbb{C}$ or the real numbers $\mathbb{R}$. Let $(V,\rangle \cdot, \cdot\rangle)$ be a (nondegenerate) symplectic space, and let $(W,(\cdot, \cdot))$ be a quadratic space over $F$. The tensor product $V \otimes W$ is a symplectic space, with the form $\langle\langle\cdot, \cdot\rangle\rangle$ defined by

$$
\langle e \otimes v, f \otimes w\rangle=\langle e, f\rangle(v, w) \quad \text { for } e, f \in V \text { and } v, w \in W .
$$

The pair of groups $(\operatorname{Sp}(V), \mathrm{O}(W))$ is called a reductive dual pair [Howe 1989].

Let $\varphi_{1}: V \otimes W \rightarrow \operatorname{Hom}(V, W)$ be the vector space isomorphism given by $\varphi_{1}(v \otimes w)(x):=\langle v, x\rangle w$, and let $\varphi_{2}: V \otimes W \rightarrow \operatorname{Hom}(W, V)$ be the isomorphism given by $\varphi_{2}(v \otimes w)(y):=(w, y) v$. Define the maps $\tau_{1}: V \otimes W \rightarrow \operatorname{End}(V)$ by $\tau_{1}(\gamma):=\varphi_{2}(\gamma) \circ \varphi_{1}(\gamma)$, and $\tau_{2}: V \otimes W \rightarrow \operatorname{End}(W)$ by $\tau_{2}(\gamma):=\varphi_{1}(\gamma) \circ \varphi_{2}(\gamma)$. It turns out that the image of $\tau_{1}$ is in $\mathfrak{s p}(V)$ and the image of $\tau_{2}$ is in $\mathfrak{s o}(W)$. The maps $\tau_{1}$ and $\tau_{2}$ are called the moment maps [Kazhdan et al. 1978].

An element $X \in \mathfrak{s p}(V)$ is said to correspond to an element $X^{\prime} \in \mathfrak{s o}(W)$ if $\tau_{1}^{-1}(X) \cap \tau_{2}^{-1}\left(X^{\prime}\right)$ is not empty. An orbit $\mathbb{O}$ in $\mathfrak{s p}(V)$ is said to correspond to an orbit $\mathcal{O}^{\prime}$ in $\mathfrak{s o}(W)$ if there is an element in $O^{O}$ that corresponds to an element in $\mathcal{O}^{\prime}$. Suppose that $\mathbb{O}$ is an orbit in $\mathfrak{s p}(V)$ corresponding to an orbit $\mathbb{O}^{\prime}$ in $\mathfrak{s o}(W)$. It is known that $\mathbb{O}$ is nilpotent if and only if $O^{\prime}$ is nilpotent. On the correspondence of nilpotent orbits, A. Daszkiewicz, W. Kraśkiewicz and T. Przebinda [1997] proved the next theorem:

MSC2000: primary 22E60; secondary 22E47, 22E30.

Keywords: orbit correspondence, nilpotent orbit, Young diagram. 
Theorem A. Let $F=\mathbb{C}$. Let $\tau_{1}: V \otimes_{F} W \rightarrow \mathfrak{s p}(V)$ and $\tau_{2}: V \otimes_{F} W \rightarrow \mathfrak{s o}(W)$ be the moment maps. Let 0 and 2 be nilpotent orbits in $\mathfrak{s p}(V)$ and $\mathfrak{s o}(W)$ contained in the images of $\tau_{1}$ and $\tau_{2}$, respectively. There are unique nilpotent orbits $\mathbb{O}^{\prime} \subseteq \mathfrak{s o}(W)$ and $\mathscr{2}^{\prime} \subseteq \mathfrak{s p}(V)$ such that $\tau_{2}\left(\tau_{1}^{-1}(\overline{\mathbb{O}})\right)=\overline{\mathbb{O}^{\prime}}$ and $\tau_{1}\left(\tau_{2}^{-1}(\overline{\mathscr{Q}})\right)=\overline{\mathscr{Q}}^{\prime}$, where $\overline{\mathscr{P}}$ denotes the closure of a nilpotent orbit $\mathscr{P}$.

In the theorem of 4.1, we give a slightly more precise form of this theorem, which coincides with [Daszkiewicz et al. 1997, Theorem 5.2]. Our main theorem is the analogous result for real orbit correspondences:

Theorem B. Suppose $F=\mathbb{R}$. Let $\tau_{1}: V \otimes_{F} W \rightarrow \mathfrak{s p}(V)$ and $\tau_{2}: V \otimes_{F} W \rightarrow \mathfrak{s o}(W)$ be the moment maps. Let $\mathcal{O}$ and 2 be nilpotent orbits in $\mathfrak{s p}(V)$ and $\mathfrak{s o}(W)$ contained in the images of $\tau_{1}$ and $\tau_{2}$, respectively. Then, $\tau_{2}\left(\tau_{1}^{-1}(\overline{0})\right)$ and $\left.\tau_{1}\left(\tau_{2}^{-1}(\overline{2})\right)\right)$ are each the union of at most two closures of nilpotent orbits in $\mathfrak{s o}(W)$ and $\mathfrak{s p}(V)$ ), respectively.

The motivation for the result is this: Suppose $G$ is a real reductive group with Lie algebra $\mathfrak{g}_{0}$. Let $\mathfrak{g}:=\mathfrak{g}_{0} \otimes_{\mathbb{R}} \mathbb{C}$ be the complexified Lie algebra of the complexified group $G_{\mathbb{C}}$ of $G$. Let $K$ be a maximal compact subgroup of $G$. Given an irreducible admissible representation $(\pi, E)$ of $G$, we can attach to it a union of nilpotent coadjoint orbits of $G_{\mathbb{C}}$ in the dual space $\mathfrak{g}^{*}$ of $\mathfrak{g}$, as follows: If $E^{K}$ is the subspace of $K$-finite vectors of $E$, then $E^{K}$ is an irreducible $(\mathfrak{g}, K)$-module. Denote the action of $\mathfrak{g}$ on $E^{K}$ by $\pi$ still. Let $I_{\pi}$ be the annihilator of $E^{K}$ in the universal enveloping algebra $U(\mathfrak{g})$ of $\mathfrak{g}$. The latter has a natural grading, and the associated graded algebra $\operatorname{gr}(U(\mathfrak{g}))$ is isomorphic to the algebra of polynomials on $\mathfrak{g}^{*}$. Let $\mathscr{V}\left(I_{\pi}\right)$ denote the variety in $\mathfrak{g}^{*}$ defined by the ideal $\operatorname{gr}\left(I_{\pi}\right)$ of $\operatorname{gr}(U(\mathfrak{g}))$. It is known that $\mathscr{V}\left(I_{\pi}\right)$ is a finite union of nilpotent orbits in $\mathfrak{g}^{*}$ and by [Collingwood and McGovern 1993; Joseph 1985] is the closure of a single nilpotent orbit. The variety $\mathscr{V}\left(I_{\pi}\right)$ is called the associated variety of the annihilator, in the universal enveloping algebra, of the Harish-Chandra module of the representation $(\pi, E)$. By a result of R. Howe [1989], there is a one-to-one correspondence between some irreducible admissible representations of the metaplectic cover $\widetilde{\mathrm{Sp}}(V)$ and some irreducible admissible representations of $\widetilde{\mathrm{O}}(W)$. Therefore, one expects a relation between the correspondence of coadjoint orbits and the correspondence of irreducible representations. Some results have been established in [Adams 1983; 1987; Przebinda 1993].

Theorem B might be interesting for studying representations of real groups by using finer orbit structures, such as wave front sets or characteristic varieties. In fact, this result is used in [He 2003; He 2005].

The content of this paper is as follows: In Sections 2 and 3, we summarize some well-known results on nilpotent orbits in $\mathfrak{s p}(V)$ and $\mathfrak{s o}(W)$. We describe some special realization of the Lie algebras $\mathfrak{s p}(V)$ and $\mathfrak{s o}(W)$, and the parametrization of 
their nilpotent orbits by some special kinds of partitions. In Section 4, we describe the results of complex orbit correspondences; the proofs are omitted, since the results in this section are already known from [Daszkiewicz et al. 1997]. In Section 5, we describe the basic rules for determining the real orbit correspondence. We state our main theorem in Section 6. The construction is somewhat subtle, although the idea is in fact simple. The proof of the main theorem is in the final Section 7.

\section{Signed partitions and signed Young diagrams}

2.1. Partitions. For nonnegative integers $r_{i}$, let $\boldsymbol{d}:=\bigoplus_{i=1}^{k}\left[r_{i}\right]$ denote the partition $\left[r_{1}, r_{2}, \ldots, r_{k}\right]$, where $r_{1} \geq r_{2} \geq \cdots \geq r_{k}$, and let $|\boldsymbol{d}|:=\sum_{i=1}^{k} r_{i}$ denote the size of $\boldsymbol{d}$. We may use $k[r]$ to denote $[r] \oplus[r] \oplus \cdots \oplus[r]$ (with $k$ terms). Suppose that $\boldsymbol{d}^{1}:=\bigoplus_{i=1}^{k_{1}}\left[r_{i}^{1}\right]$ and $\boldsymbol{d}^{2}:=\bigoplus_{i=1}^{k_{2}}\left[r_{i}^{2}\right]$ are two partitions of the same size. We write

$$
\boldsymbol{d}^{1} \leq \boldsymbol{d}^{2} \quad \text { if } \quad \sum_{i=1}^{j} r_{i}^{1} \leq \sum_{i=1}^{j} r_{i}^{2} \quad \text { for each } j \geq 1 .
$$

This defines a partial ordering on the set of partitions of same size.

2.2. Symplectic and orthogonal partitions. A partition $\bigoplus_{i=1}^{k} \alpha_{i}\left[r_{i}\right]$ of pairwise distinct $r_{i}$ is symplectic if $\alpha_{i}$ is even whenever $r_{i}$ is odd. A symplectic partition is said to be simple if it is of the form [2r] or 2[2r-1] for some positive integer $r$. It is clear that a symplectic partition is a sum of simple symplectic partitions.

A partition $\bigoplus_{i=1}^{k} \alpha_{i}\left[r_{i}\right]$ is orthogonal if $\alpha_{i}$ is even whenever $r_{i}$ is even. An orthogonal partition is simple if it is of the form [2r-1] or 2[2r] for some positive integer $r$. An orthogonal partition is a sum of simple orthogonal partitions.

2.3. Signed partitions and signed Young diagrams. A signed partition is a sum $\bigoplus_{i=1}^{k}\left[r_{i}\right]^{\bullet}$ of symbols $\left[r_{i}\right]^{\bullet}$, where $r_{i}$ is a nonnegative integer and $\bullet$ is either plus or minus.

The signed partitions $[r]^{-}$and $[r]^{+}$can be presented as rows of $r$ with boxes labeled alternatingly by plus and minus signs, ending with $\boxminus$ and $\boxplus$, respectively. For example, $[2 r]^{-}$has the presentation

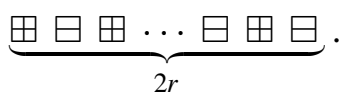

By convention, we set $[0]^{+}=[0]^{-}:=\varnothing$. The signed partitions $[r]^{+}$and $[r]^{-}$are respectively called positive and negative rows.

A presentation of a signed partition is called a signed Young diagram if the sizes of the rows are nonincreasing. Two signed Young diagrams of a signed partition are not regarded as the same if they differ by exchanging two rows of the same 
length but opposite parity. For example, the signed Young diagrams

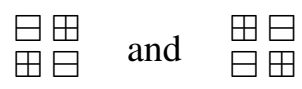

are regarded as different, although they are associated to the same signed partition $[2]^{+} \oplus[2]^{-}$. Giving a signed Young diagram of a signed partition is equivalent to defining a total order on the set of rows of the signed partition. For example, the first signed Young diagram above gives the order $[2]^{+}>[2]^{-}$, while the second Young diagram gives the order $[2]^{-}>[2]^{+}$. Therefore, we will use the notation $(\boldsymbol{d}, Q)$ to denote a signed Young diagram of a signed partition $\boldsymbol{d}$, where $Q$ is an order on the set of rows of $\boldsymbol{d}$.

2.4. Signature of a signed partition. If $\boldsymbol{d}:=\bigoplus_{i=1}^{k}\left[r_{i}\right]^{*}$ is a signed partition, the signature of $\boldsymbol{d}$ is the ordered pair defined by

$$
\operatorname{sgn}(\boldsymbol{d}):=\sum_{i=1}^{k} \operatorname{sgn}\left(\left[r_{i}\right]^{*}\right)
$$

with $\operatorname{sgn}\left([r]^{+}\right):=(\lfloor(r+1) / 2\rfloor,\lfloor r / 2\rfloor)$ and $\operatorname{sgn}\left([r]^{-}\right):=(\lfloor r / 2\rfloor,\lfloor(r+1) / 2\rfloor)$, where $\lfloor a\rfloor$ stands for the largest integer not greater than $a$, and $(p, q)+(r, s)$ is defined to be $(p+r, q+s)$. That is, the first (respectively, second) component of the signature is the number of plus (respectively, minus) boxes in any presentation of a signed partition.

2.5. Derivatives of signed partitions. Define the derivatives of a partition or of a signed partition as follows: Let

$$
[r]^{\prime}:=[r-1], \quad\left([r]^{+}\right)^{\prime}:=[r-1]^{+}, \quad\left([r]^{-}\right)^{\prime}:=[r-1]^{-} .
$$

Define $\boldsymbol{d}^{\prime}:=\bigoplus_{i=1}^{k}\left(\left[r_{i}\right]^{*}\right)^{\prime}$ if $\boldsymbol{d}=\bigoplus_{i=1}^{k}\left[r_{i}\right]^{*}$. Let $\boldsymbol{d}^{(0)}:=\boldsymbol{d}$, and $\boldsymbol{d}^{(k)}:=\left(\boldsymbol{d}^{(k-1)}\right)^{\prime}$ for $k \geq 1$.

For two pairs of positive integers, the order $(p, q) \leq(r, s)$ stands for $p \leq r$ and $q \leq s$. Suppose that $\boldsymbol{d}_{1}$ and $\boldsymbol{d}_{2}$ are two signed partitions of the same signature. We say that $\boldsymbol{d}_{1} \leq \boldsymbol{d}_{2}$ if $\operatorname{sgn}\left(\boldsymbol{d}_{1}^{(k)}\right) \leq \operatorname{sgn}\left(\boldsymbol{d}_{2}^{(k)}\right)$ for all $k \geq 0$.

2.6. Symplectic and orthogonal signed partitions. A signed partition $\boldsymbol{d}$ is simple symplectic if $\boldsymbol{d}$ is equal to $[2 r]^{+},[2 r]^{-}$, or $[2 r-1]^{+} \oplus[2 r-1]^{-}$for some positive integer $r$. The signed partition $[2 r-1]^{+} \oplus[2 r-1]^{-}$is called a double odd block, with $[2 r-1]^{+}$called the partner of $[2 r-1]^{-}$and vice versa. A sum of simple symplectic signed partitions is a symplectic signed partition.

We say a signed partition $\boldsymbol{d}$ is simple orthogonal if $\boldsymbol{d}$ is $[2 r-1]^{+},[2 r-1]^{-}$ or $[2 r]^{+} \oplus[2 r]^{-}$for some positive integer $r$. A sum of simple orthogonal signed partitions is an orthogonal signed partition. 


\section{Nilpotent orbits and moment maps}

3.1. Nilpotent orbits in a symplectic Lie algebra. Let $F$ be the field $\mathbb{C}$ or $\mathbb{R}$. Let $(V,\langle\cdot, \cdot\rangle)$ be a $2 n$-dimensional symplectic space over $F$. The Lie algebra $\mathfrak{s p}(V)$ consists of elements $X \in \operatorname{End}_{F}(V)$ such that $\langle X . v, w\rangle+\langle v, X . w\rangle=0$ for all $v, w \in V$. The Lie algebra $\mathfrak{s p}(V)$ can be realized as the space $\mathrm{S}^{2}(V)$ of degree two elements in the symmetric algebra of $V$, with the Lie bracket given by

$$
\left[e f, e^{\prime} f^{\prime}\right]:=\left\langle e^{\prime}, e\right\rangle f f^{\prime}+\left\langle f^{\prime}, e\right\rangle f e^{\prime}+\left\langle e^{\prime}, f\right\rangle e f^{\prime}+\left\langle f^{\prime}, f\right\rangle e e^{\prime}
$$

for $e, e^{\prime}, f, f^{\prime} \in V$. The action of $\mathrm{S}^{2}(V)$ on $V$ is given by

$$
(e f)(x):=\langle x, e\rangle f+\langle x, f\rangle e
$$

for $e, f, x \in V$. Details can be found in [Howe 1989].

It is well known that, when $F=\mathbb{R}$, the nilpotent orbits of the symplectic group $\operatorname{Sp}(V)$ in $\mathfrak{s p}(V)$ are parametrized by the set of symplectic signed partitions of size $2 n$ [Collingwood and McGovern 1993]. We can take

$$
e_{1} f_{2}+\cdots+e_{r-1} f_{r}+e_{r} e_{r}
$$

as representative of the nilpotent orbit corresponding to the simple signed partition $[2 r]^{-}$, where $\left\{e_{1}, \ldots, e_{r}, f_{1}, \ldots, f_{r}\right\}$ is a self-dual basis (that is, $\left\langle e_{i}, f_{j}\right\rangle=\delta_{i j}$ ) of a $2 r$-dimensional symplectic subspace of $V$. Similarly, we can take

$$
e_{1} f_{2}+\cdots+e_{r-1} f_{r}-e_{r} e_{r}
$$

as representative of the nilpotent orbit corresponding to $[2 r]^{+}$, and

$$
e_{1} f_{2}+\cdots+e_{2 r-2} f_{2 r-1}
$$

as representative of the nilpotent orbit corresponding to $[2 r-1]^{+} \oplus[2 r-1]^{-}$.

3.2. Nilpotent orbits in an orthogonal Lie algebra. Suppose $(W,(\cdot, \cdot))$ is an $m$ dimensional quadratic space over $F$. The Lie algebra $\mathfrak{s o}(W)$ can be realized as the space $\bigwedge^{2}(W)$ of degree two elements in the exterior algebra of $W$, with the Lie bracket given by

$\left[v \wedge w, v^{\prime} \wedge w^{\prime}\right]:=\left(v, v^{\prime}\right) g w \wedge w^{\prime}+\left(v, w^{\prime}\right) w \wedge v^{\prime}+\left(w, v^{\prime}\right) g v \wedge w^{\prime}+\left(w, w^{\prime}\right) v \wedge v^{\prime}$ for $v, v^{\prime}, w, w^{\prime} \in W$. The Lie algebra $\wedge^{2}(W)$ acts on $W$ by

$$
(v \wedge w)(y):=(y, w) v-(y, v) w \text { for } v, w, y \in W .
$$

It is well known that, when $F=\mathbb{R}$, the nilpotent orbits of the orthogonal group $\mathrm{O}(W)$ in $\mathfrak{s o}(W)$ are parametrized by the set of orthogonal signed partitions with 
signature equal to that of the form $(\cdot, \cdot)$ [Collingwood and McGovern 1993]. We can take

$$
v_{1} \wedge w_{2}+v_{2} \wedge w_{3}+\cdots+v_{r-2} \wedge w_{r-1}+v_{r-1} \wedge z
$$

as representative of the nilpotent orbit corresponding to the simple orthogonal signed partition $[2 r-1]^{+}$, where $\left\{z, v_{1}, \ldots, v_{r-1}, w_{1}, \ldots, w_{r-1}\right\}$ is a basis of a $(2 r-1)$-dimensional orthogonal subspace of signature $(r, r-1)$ such that

$$
\left(v_{i}, w_{j}\right)=\delta_{i j}, \quad\left(v_{i}, v_{j}\right)=\left(w_{i}, w_{j}\right)=0, \quad\left(v_{i}, z\right)=\left(w_{i}, z\right)=0, \quad(z, z)=1 .
$$

Similarly, we can take $v_{1} \wedge w_{2}+v_{2} \wedge w_{3}+\cdots+v_{r-2} \wedge w_{r-1}+v_{r-1} \wedge z^{\prime}$ as representative of the nilpotent orbit corresponding to $[2 r-1]^{-}$, where $\left(z^{\prime}, z^{\prime}\right)=-1$, and take $v_{1} \wedge w_{2}+v_{2} \wedge w_{3}+\cdots+v_{2 r-1} \wedge w_{2 r}$ as representative of the nilpotent orbit corresponding to $[2 r]^{+} \oplus[2 r]^{-}$.

3.3. Derivatives and the ordering of orbits. Let $\mathrm{O}_{1}$ and $\mathrm{O}_{2}$ be two nilpotent orbits in $\mathfrak{s p}(V)$ (or in $\mathfrak{s o}(W)$ ), with corresponding signed partitions $\boldsymbol{d}_{1}$ and $\boldsymbol{d}_{2}$, respectively. We define $\mathrm{O}_{1} \leq \mathrm{O}_{2}$ if $\mathrm{O}_{1} \subset \overline{\mathrm{O}}_{2}$, the closure of $\mathrm{O}_{2}$.

Proposition [Djoković 1981]. We have $\mathbb{O}_{1} \leq \mathbb{O}_{2}$ if and only if $\operatorname{sgn}\left(\boldsymbol{d}_{1}^{(k)}\right) \leq \operatorname{sgn}\left(\boldsymbol{d}_{2}^{(k)}\right)$ for every $k \geq 0$.

3.4. Moment maps. Consider the vector space isomorphisms

$$
\begin{aligned}
& \varphi_{1}: V \otimes W \rightarrow \operatorname{Hom}(V, W), \quad \varphi_{1}(v \otimes w)(x):=\langle v, x\rangle w, \\
& \varphi_{2}: V \otimes W \rightarrow \operatorname{Hom}(W, V), \quad \varphi_{2}(v \otimes w)(y):=(w, y) v
\end{aligned}
$$

for $v, x \in V$ and $w, y \in W$, and define the moment maps

$$
\begin{array}{ll}
\tau_{1}: V \otimes W \rightarrow \operatorname{End}(V), & \tau_{1}(\gamma):=\varphi_{2}(\gamma) \circ \varphi_{1}(\gamma), \\
\tau_{2}: V \otimes W \rightarrow \operatorname{End}(W), & \tau_{2}(\gamma):=\varphi_{1}(\gamma) \circ \varphi_{2}(\gamma) .
\end{array}
$$

It is known that $\tau_{1}(V \otimes W) \subseteq \mathfrak{s p}(V)$ and $\tau_{2}(V \otimes W) \subseteq \mathfrak{s o}(W)$. It is not difficult to check that

$$
\tau_{1}(\gamma)=\sum_{i, j}\left(\eta_{i}, \eta_{j}\right) \varepsilon_{i} \varepsilon_{j} \quad \text { and } \quad \tau_{2}(\gamma)=\sum_{i, j}\left\langle\varepsilon_{i}, \varepsilon_{j}\right\rangle \eta_{i} \wedge \eta_{j}
$$

when $\gamma:=\sum_{i} \varepsilon_{i} \otimes \eta_{i} \in V \otimes W$ with $\varepsilon_{i} \in V$ and $\eta_{i} \in W$, under the realization $\mathfrak{s p}(V)=\mathrm{S}^{2}(V)$ and $\mathfrak{s o}(W)=\bigwedge^{2}(W)$.

\section{The orbit correspondences for complex reductive dual pairs}

In this section, we assume that $F=\mathbb{C}$. Let $V$ be a $2 n$-dimensional complex symplectic space and $W$ be an $m$-dimensional complex quadratic space. Let $\tau_{1}$ and $\tau_{2}$ be the moment maps from 3.4. 
4.1. The purpose of Section 4 is, for a nilpotent orbit $O$ in $\mathfrak{s p}(V)$, to define a nilpotent orbit $\Phi(\lambda(\mathcal{O}))$ in $\mathfrak{s o}(W)$ such that the following theorem holds.

Theorem. Let $\mathbb{O}$ be a nilpotent orbit in $\mathfrak{s p}(V)$ that is contained in the image of $\tau_{1}$. Then $\tau_{2}\left(\tau_{1}^{-1}(\overline{\mathbb{O}})\right)=\overline{\Phi(\lambda(\mathbb{O}))}$.

4.2. Let $\boldsymbol{d}:=\bigoplus_{i=1}^{k} \alpha_{i}\left[r_{i}\right]$ be a symplectic partition of size $2 n$, with $r_{1}>r_{2}>\cdots>$ $r_{k}>0$. Define the numbers $r_{i}^{1}=r_{i}^{2}=\cdots=r_{i}^{\alpha_{i}}:=r_{i}$ for each $i=1,2, \ldots, k$, so we may write

$$
\boldsymbol{d}=\bigoplus_{i=1}^{k} \bigoplus_{j=1}^{\alpha_{i}}\left[r_{i}^{j}\right]
$$

Let $\alpha:=\sum_{i=1}^{k} \alpha_{i}$ be the number of rows of $\boldsymbol{d}$. We define

$$
\varphi(\boldsymbol{d}):= \begin{cases}\bigoplus_{i=1}^{k} \bigoplus_{j=1}^{\alpha_{i}}\left[r_{i}^{j}-1\right] \oplus(\alpha+m-2 n)[1] & \text { if }-\alpha \leq m-2 n, \\ {[0]} & \text { otherwise. }\end{cases}
$$

It is not difficult to see that the partition $\varphi(\boldsymbol{d})$ is either [0] or an orthogonal partition of size $m$. Define

$$
\varphi(\mathcal{O}):= \begin{cases}\mathcal{O}_{\varphi(\boldsymbol{d})} & \text { if } \varphi(\boldsymbol{d}) \neq[0] \\ \varnothing & \text { if } \varphi(\boldsymbol{d})=[0]\end{cases}
$$

that is, if it is nonempty, $\varphi(\mathcal{O})$ is a nilpotent orbit in $\mathfrak{s o}(W)$.

4.3. We define two partitions $\psi_{1}(\boldsymbol{d})$ and $\psi_{2}(\boldsymbol{d})$ according to the following cases:

(i) Suppose that $\alpha<m-2 n$; define

$$
\psi_{1}(\boldsymbol{d}):=\bigoplus_{i=1}^{k} \alpha_{i}\left[r_{i}\right] \oplus \alpha[1] \quad \text { and } \quad \psi_{2}(\boldsymbol{d}):=(m-2 n-\alpha)[1]
$$

(ii) Suppose that $0 \leq m-2 n \leq \alpha$; define

$$
\psi_{1}(\boldsymbol{d}):=\bigoplus_{i=1}^{k} \alpha_{i}\left[r_{i}\right] \oplus(m-2 n)[1] \quad \text { and } \quad \psi_{2}(\boldsymbol{d}):=[0] .
$$

(iii) Suppose that $-\alpha \leq m-2 n<0$. Let $x_{0}$ and $y_{0}$ be the indices such that $r_{x_{0}}^{y_{0}}$ be the $(2 n-m)$-th number from the smallest in the ordered sequence

(4-3) $r_{1}^{1} \geq \cdots \geq r_{1}^{\alpha_{1}}>r_{2}^{1} \geq \cdots \geq r_{2}^{\alpha_{2}}>\cdots>r_{k-1}^{1} \geq \cdots \geq r_{k-1}^{\alpha_{k-1}}>r_{k}^{1} \geq \cdots \geq r_{k}^{\alpha_{k}}$, 
that is, choose $x_{0}$ and $y_{0}$ so that $\alpha_{x_{0}}-y_{0}+1+\sum_{i=x_{0}+1}^{k} \alpha_{i}=2 n-m$. Define

$$
\begin{aligned}
& \psi_{1}(\boldsymbol{d}):=\bigoplus_{i=1}^{x_{0}-1} \alpha_{i}\left[r_{i}\right] \oplus\left(y_{0}-1\right)\left[r_{x_{0}}\right] \quad \text { and } \\
& \psi_{2}(\boldsymbol{d}):=\left(\alpha_{x_{0}}-y_{0}+1\right)\left[r_{x_{0}}-1\right] \oplus \bigoplus_{i=x_{0}+1}^{k} \alpha_{i}\left[r_{i}-1\right] .
\end{aligned}
$$

(iv) Suppose that $m-2 n<-\alpha$; define $\psi_{1}(\boldsymbol{d})=\psi_{2}(\boldsymbol{d}):=[0]$.

Note that the definitions of $\psi_{1}(\boldsymbol{d})$ and $\psi_{2}(\boldsymbol{d})$ depend on the dimensions $m$ and $2 n$.

4.4. For $\psi_{1}(\boldsymbol{d})$ as given in 4.3 , we define the element $\Phi^{*}\left(\psi_{1}(\boldsymbol{d})\right)$. If $\psi_{1}(\boldsymbol{d})=[0]$, we define $\Phi^{*}\left(\psi_{1}(\boldsymbol{d})\right):=[0]$. Assume next that $\psi_{1}(\boldsymbol{d}) \neq[0]$. Suppose that

$$
\psi_{1}(\boldsymbol{d})=\bigoplus_{i=1}^{l} \bigoplus_{j=1}^{\beta_{i}}\left[f_{i}^{j}\right]
$$

where the numbers $f_{i}^{j}$ are ordered as

$$
\begin{aligned}
f_{1}^{1}=\cdots=f_{1}^{\beta_{1}}>f_{2}^{1}=\cdots= & f_{2}^{\beta_{2}}>\cdots \\
& >f_{l-1}^{1}=\cdots=f_{l-1}^{\beta_{l-1}}>f_{l}^{1}=\cdots=f_{l}^{\beta_{l}}>0 .
\end{aligned}
$$

Let $\beta:=\sum_{i=1}^{l} \beta_{i}$ be the number of rows of $\psi_{1}(\boldsymbol{d})$. We define the number $s_{x}^{y}$ according to the following cases:

(i) Suppose that $\beta$ is even, and let $f_{x_{2}}^{y_{2}} \geq f_{x_{1}}^{y_{1}}$ be the two median numbers in (4-4).

(a) If $f_{x_{1}}^{y_{1}}=f_{x_{2}}^{y_{2}}$ are odd and $y_{1}$ is even, we define $s_{x}^{y}$ by

$$
s_{x}^{y}:= \begin{cases}f_{x}^{y}+1 & \text { if either } x=x_{2} \text { and } y<y_{2}, \text { or } x<x_{2} ; \\ f_{x}^{y} & \text { if either } x=x_{1} \text { and } y=y_{1} \text {, or } x=x_{2} \text { and } y=y_{2} ; \\ f_{x}^{y}-1 & \text { if either } x=x_{1} \text { and } y>y_{1}, \text { or } x>x_{1} .\end{cases}
$$

(b) Otherwise, we define

$$
s_{x}^{y}:= \begin{cases}f_{x}^{y}+1 & \text { if either } x=x_{2} \text { and } y \leq y_{2}, \text { or } x<x_{2} \\ f_{x}^{y}-1 & \text { if either } x=x_{1} \text { and } y \geq y_{1} \text {, or } x>x_{1}\end{cases}
$$

(ii) Suppose that $\beta$ is odd, and let $f_{x_{3}}^{y_{3}} \geq f_{x_{2}}^{y_{2}} \geq f_{x_{1}}^{y_{1}}$ be the three median numbers in (4-4).

(a) If $f_{x_{2}}^{y_{2}}=f_{x_{3}}^{y_{3}}$ are odd and $y_{2}$ is even, we define

$$
s_{x}^{y}:= \begin{cases}f_{x}^{y}+1 & \text { if either } x=x_{3} \text { and } y<y_{3}, \text { or } x<x_{3} ; \\ f_{x}^{y} & \text { if either } x=x_{2} \text { and } y=y_{2}, \text { or } x=x_{3} \text { and } y=y_{3} ; \\ f_{x}^{y}-1 & \text { if either } x=x_{1} \text { and } y \geq y_{1}, \text { or } x>x_{1} .\end{cases}
$$


(b) Otherwise, we define

$$
s_{x}^{y}:= \begin{cases}f_{x}^{y}+1 & \text { if either } x=x_{3} \text { and } y \leq y_{3}, \text { or } x<x_{3} \\ f_{x}^{y}-1 & \text { if either } x=x_{2} \text { and } y \geq y_{2}, \text { or } x>x_{2} .\end{cases}
$$

Then, we define

$$
\begin{aligned}
\Phi^{*}\left(\psi_{1}(\boldsymbol{d})\right) & := \begin{cases}\bigoplus_{i=1}^{l} \bigoplus_{j=1}^{\beta_{i}}\left[s_{i}^{j}\right] & \text { for case (i.a) and (i.b) } \\
\bigoplus_{i=1}^{l} \bigoplus_{j=1}^{\beta_{i}}\left[s_{i}^{j}\right] \oplus[1] & \text { for case (ii.a) and (ii.b) }\end{cases} \\
\Phi(\boldsymbol{d}) & :=\Phi^{*}\left(\psi_{1}(\boldsymbol{d})\right) \oplus \psi_{2}(\boldsymbol{d}) .
\end{aligned}
$$

The partition $\Phi(\boldsymbol{d})$ is either [0] or an orthogonal partition of size $m$. Define

$$
\Phi(\mathcal{O}):= \begin{cases}\mathcal{O}_{\Phi(\boldsymbol{d})} & \text { if } \Phi(\boldsymbol{d}) \neq[0], \\ \varnothing & \text { if } \Phi(\boldsymbol{d})=[0],\end{cases}
$$

that is, $\Phi(\mathbb{O})$ is a nilpotent orbit in $\mathfrak{s o}(W)$ if it is nonempty.

4.5. Example. Suppose that $2 n=24, m=25$, and $\boldsymbol{d}=[6] \oplus 2[5] \oplus[4] \oplus 2[2]$; that is, $r_{1}^{1}=6, r_{2}^{1}=5, r_{2}^{2}=5, r_{3}^{1}=4, r_{4}^{1}=2$, and $r_{4}^{2}=2$. We have $\alpha=1+2+1+2=6$ and $m-2 n=25-24=1$, so we are in case (ii) of 4.3. Thus, $\psi_{2}(\boldsymbol{d})=[0]$ and $\psi_{1}(\boldsymbol{d})=[6] \oplus 2[5] \oplus[4] \oplus 2[2] \oplus[1]$. Now, $\alpha+(m-2 n)=7$ is odd, and the three median numbers of sequence Equation (4-4) are $5>4>2$. We are in case (ii.b) of 4.4 and, hence, $s_{1}^{1}=7, s_{2}^{1}=6, s_{2}^{2}=6, s_{3}^{1}=3, s_{4}^{1}=1, s_{4}^{2}=1$, and $s_{5}^{1}=0$. Thus, $\Phi(\boldsymbol{d})=\Phi^{*}\left(\psi_{1}(\boldsymbol{d})\right)=[7] \oplus 2[6] \oplus[3] \oplus 3[1]$ and $\varphi(\boldsymbol{d})=[5] \oplus 2[4] \oplus[3] \oplus 9[1]$.

4.6. Proposition. If $\mathcal{O}$ is a nilpotent orbit contained in the image of $\tau_{1}$, then $\Phi(0)$ is the unique maximal element and $\varphi(\mathcal{O})$ is the unique minimal element in the set of nilpotent orbits in $\tau_{2}\left(\tau_{1}^{-1}(\mathbb{O})\right)$.

4.7. Suppose that we are not in the case (iv) of 4.3. We want to define a new partition $\lambda(\boldsymbol{d})$ of size $2 n$. If $m-2 n \geq \alpha$, we define $\lambda(\boldsymbol{d}):=\boldsymbol{d}$. Next, we suppose that $m-2 n<\alpha$. Define a row $\left[r_{x_{0}}^{y_{0}}\right]$ as follows: Let $\left[r_{x_{0}}^{y_{0}}\right]$ be the smallest element in the ordered sequence (4-3) satisfying the condition

$$
\sum_{i=x_{0}+1}^{k} \alpha_{i} r_{i}+\left(\alpha_{x_{0}}-y_{0}+1\right) r_{x_{0}}+(m-2 n) \geq y_{0}-1+\sum_{i=1}^{x_{0}-1} \alpha_{i},
$$

where $x_{0}$ is some number between 1 and $k$, and $y_{0}$ is between 1 and $\alpha_{x_{0}}$. Define

$$
\begin{aligned}
& \sigma:=\sum_{i=x_{0}+1}^{k} \alpha_{i} r_{i}+\left(\alpha_{x_{0}}-y_{0}+1\right) r_{x_{0}}+(m-2 n)-\left(y_{0}-1+\sum_{i=1}^{x_{0}-1} \alpha_{i}\right), \\
& \delta:=y_{0}-1+\sum_{i=1}^{x_{0}-1} \alpha_{i}-(m-2 n) .
\end{aligned}
$$


It is clear that $0 \leq \sigma<r_{x_{0}}^{y_{0}}$ and $0 \leq \delta$. We define $\lambda(\boldsymbol{d})$ according to the following cases:

(i) If $r_{x_{0}}^{y_{0}}$ is even or $y_{0}$ is odd, we define

$$
\lambda(\boldsymbol{d}):=\boldsymbol{e} \oplus \begin{cases}{[\sigma-1] \oplus(\delta+1)[1],} & \text { if } \sigma \text { is odd, } \\ \delta[1] & \text { if } \sigma=0, \\ {[\sigma-2] \oplus(\delta+2)[1]} & \text { if } \sigma \text { is positive and even, }\end{cases}
$$

where

$$
\boldsymbol{e}:=\bigoplus_{i=1}^{x_{0}-1} \bigoplus_{j=1}^{\alpha_{i}}\left[r_{i}^{j}\right] \oplus \bigoplus_{j=1}^{y_{0}-1}\left[r_{x_{0}}^{j}\right]
$$

(ii) If $r_{x_{0}}^{y_{0}}$ is odd and $y_{0}$ is even, we define

$$
\lambda(\boldsymbol{d}):=\boldsymbol{e}^{\prime} \oplus \begin{cases}{[\sigma-1] \oplus(\delta+2)[1],} & \text { if } \sigma \text { is odd, } \\ {[\sigma] \oplus(\delta+1)[1],} & \text { if } \sigma \text { is even, }\end{cases}
$$

where

$$
\boldsymbol{e}^{\prime}:=\bigoplus_{i=1}^{x_{0}-1} \bigoplus_{j=1}^{\alpha_{i}}\left[r_{i}^{j}\right] \oplus \bigoplus_{j=1}^{y_{0}-2}\left[r_{x_{0}}^{j}\right] \oplus\left[r_{x_{0}}^{y_{0}-1}-1\right] .
$$

It is not difficult to see that $\lambda(\boldsymbol{d})$ is a symplectic partition of size $2 n$ and $\lambda(\boldsymbol{d}) \leq \boldsymbol{d}$. Finally, we define

$$
\lambda\left(\mathscr{O}_{d}\right):=\mathscr{O}_{\lambda(d)} .
$$

4.8. Example. Suppose that $2 n=m=60$ and $\boldsymbol{d}=6[8] \oplus 2[6]$. Then, $m-2 n=0$, $\alpha=6+2=8, \psi_{2}(\boldsymbol{d})=[0]$ and $\psi_{1}(\boldsymbol{d})=\boldsymbol{d}$. Hence,

$$
\Phi(\boldsymbol{d})=\Phi^{*}\left(\psi_{1}(\boldsymbol{d})\right)=4[9] \oplus 2[7] \oplus 2[5] .
$$

Now, $r_{1}^{1}=r_{1}^{2}=\cdots=r_{1}^{6}=8, r_{2}^{1}=r_{2}^{2}=6, \alpha_{1}=6, \alpha_{2}=2$ and $k=2$. Therefore, $r_{x_{0}}^{y_{0}}=r_{2}^{1}$, that is, $x_{0}=2$ and $y_{0}=1$. Hence, $\sigma=0+(2-1+1) \cdot 6+0-(1-1+6)=6$, which is even, and $\delta=(1-1+6)-0=6$. Then, $\lambda(\boldsymbol{d})=6[8] \oplus[4] \oplus 8[1]$. The number of rows for $\lambda(\boldsymbol{d})$ is $6+1+8=15$. Hence, $\psi_{1}(\lambda(\boldsymbol{d}))=\lambda(\boldsymbol{d})$ and $\psi_{2}(\lambda(\mathbf{d}))=[0]$. Write $\psi_{1}(\lambda(\boldsymbol{d}))=\bigoplus_{i=1}^{l} \bigoplus_{j=1}^{\beta_{i}}\left[f_{i}^{j}\right]$, where $f_{1}^{1}=f_{1}^{2}=\cdots=f_{1}^{6}=8, f_{2}^{1}=4$, and $f_{3}^{1}=f_{3}^{2}=\cdots=f_{3}^{8}=1$. The number of rows of $\psi_{1}(\lambda(\boldsymbol{d}))$ is odd. So now we are in the case (ii.b) of 4.4 and, therefore, $\Phi(\lambda(\boldsymbol{d}))=\Phi^{*}\left(\psi_{1}(\lambda(\boldsymbol{d}))\right)=6[9] \oplus[5] \oplus[1]$. It is clear that $\lambda(\boldsymbol{d})<\boldsymbol{d}$, but $\Phi(\lambda(\mathbf{d}))>\Phi(\boldsymbol{d})$.

4.9. Proposition. Let 0 be a nilpotent orbit contained in the image of $\tau_{1}$. Then, $\Phi(\lambda(0))$ is the unique maximal element in the set $\{\Phi(2) \mid 2 \leq 0\}$.

\section{The rules for simple signed partitions}

From now on to the end of this paper we assume that $F=\mathbb{R}$. Let $\tau_{1}$ and $\tau_{2}$ be the maps defined in 3.4 . 
5.1. Suppose $\mathcal{O}$ is a nilpotent orbit in $\mathfrak{s p}(V)$ that is in the image of $\tau_{1}$, that $\boldsymbol{d}$ is the signed partition corresponding to $\mathcal{O}$, and that $\gamma$ is an element in $V \otimes W$ such that $\tau_{1}(\gamma)$ is in 0 . Assume $V=V^{1} \oplus \cdots \oplus V^{k}$ is an orthogonal decomposition corresponding to the Jordan blocks of the decomposition $\boldsymbol{d}=\bigoplus_{i=1}^{k} \boldsymbol{d}^{i}$, such that each $\boldsymbol{d}^{i}$ is a simple symplectic signed partition, as introduced in 2.6. Now, $\gamma$ can be written as $\left(\gamma^{1}, \ldots, \gamma^{k}\right)$, where $\gamma^{i} \in V^{i} \otimes W$, and $\tau_{1}(\gamma) \in \bigoplus_{i=1}^{k} \mathfrak{s p}\left(V^{i}\right) \subseteq \mathfrak{s p}(V)$. Write $\tau_{1}(\gamma)=\left(\pi^{1}\left(\tau_{1}(\gamma)\right), \ldots, \pi^{k}\left(\tau_{1}(\gamma)\right)\right)$ with $\pi^{i}\left(\tau_{1}(\gamma)\right) \in \mathfrak{s p}\left(V^{i}\right)$ for each $i$, and assume that

$$
\pi^{i}\left(\tau_{1}(\gamma)\right)=e_{1}^{i} f_{2}^{i}+\cdots+e_{r_{i}-1}^{i} f_{r_{i}}^{i}+e_{r_{i}}^{i} e_{r_{i}}^{i}
$$

that is, $\pi^{i}\left(\tau_{1}(\gamma)\right)$ is an element of the nilpotent orbit in $\mathfrak{s p}\left(V^{i}\right)$ corresponding to $\left[2 r_{i}\right]^{-}$, as in 3.1. Then, there is a self-dual set $S=\left\{v_{l}^{i}, w_{l}^{i}, z^{i} \mid l=1, \ldots, r_{i}-1\right\}$ and an isotropic vector $\omega^{i}$ (possibly trivial) orthogonal to $S$, such that $\gamma^{i}$ is of the form

$$
\begin{aligned}
e_{1}^{i} \otimes v_{1}^{i}+e_{2}^{i} \otimes v_{2}^{i}+\cdots & +e_{r_{i}-1}^{i} \otimes v_{r_{i}-1}^{i}+e_{r_{i}}^{i} \otimes z^{i} \\
& +f_{1}^{i} \otimes \omega^{i}+f_{2}^{i} \otimes w_{1}^{i}+\cdots+f_{r_{i}-1}^{i} \otimes w_{r_{i}-2}^{i}+f_{r_{i}}^{i} \otimes w_{r_{i}-1}^{i} .
\end{aligned}
$$

Therefore, by Equation (3-1), $\tau_{2}(\gamma)$ has a block of the form

$$
v_{1}^{i} \wedge \omega^{i}+v_{2}^{i} \wedge w_{1}^{i}+\cdots+v_{r_{i}-1}^{i} \wedge w_{r_{i}-2}^{i}+z^{i} \wedge w_{r_{i}-1}^{i} .
$$

This block corresponds to $\left[2 r_{i}+1\right]^{+}$or $\left[2 r_{i}-1\right]^{+}$, depending on whether the vector $\omega^{i}$ can be nonzero or not.

5.2. Assume that $\pi^{i}\left(\tau_{1}(\gamma)\right)=e_{1}^{i} f_{2}^{i}+\cdots+e_{r_{i}-1}^{i} f_{r_{i}}^{i}-e_{r_{i}}^{i} e_{r_{i}}^{i}$; that is, $\pi^{i}\left(\tau_{1}(\gamma)\right)$ is an element in the nilpotent orbit in $\mathfrak{s p}\left(V^{i}\right)$ corresponding to $\left[2 r_{i}\right]^{+}$. Then, there is a set $S=\left\{v_{l}^{i}, w_{l}^{i}, z^{i} \mid l=1, \ldots, r_{i}-1\right\}$ such that

$$
\left(v_{r}^{i}, w_{s}^{i}\right)=\delta_{r s}, \quad\left(v_{r}^{i}, v_{s}^{i}\right)=\left(w_{r}^{i}, w_{s}^{i}\right)=\left(v_{r}^{i}, z^{\prime i}\right)=\left(w_{r}^{i}, z^{\prime i}\right)=0, \quad\left(z^{i i}, z^{\prime i}\right)=-1
$$

for any $r, s=1, \ldots, l$ and an isotropic vector $\omega^{i}$ orthogonal to $S$, such that $\gamma^{i}$ is of the form

$$
\begin{aligned}
e_{1}^{i} \otimes v_{1}^{i}+e_{2}^{i} \otimes v_{2}^{i}+\cdots & +e_{r_{i}-1}^{i} \otimes v_{r_{i}-1}^{i}+e_{r_{i}}^{i} \otimes z^{\prime i} \\
& +f_{1}^{i} \otimes \omega^{i}+f_{2}^{i} \otimes w_{1}^{i}+\cdots+f_{r_{i}-1}^{i} \otimes w_{r_{i}-2}^{i}+f_{r_{i}}^{i} \otimes w_{r_{i}-1}^{i} .
\end{aligned}
$$

Therefore, $\tau_{2}(\gamma)$ has a block of the form

$$
v_{1}^{i} \wedge \omega^{i}+v_{2}^{i} \wedge w_{1}^{i}+\cdots+v_{r_{i}-1}^{i} \wedge w_{r_{i}-2}^{i}+z^{i} \wedge w_{r_{i}-1}^{i} .
$$

This block corresponds to $\left[2 r_{i}+1\right]^{-}$or $\left[2 r_{i}-1\right]^{-}$, depending on whether the vector $\omega^{i}$ can be nonzero or not. 
5.3. Assume now that

$$
\pi^{i}\left(\tau_{1}(\gamma)\right)=e_{1}^{i} f_{2}^{i}+\cdots+e_{2 r_{i}-2}^{i} f_{2 r_{i}-1}^{i}
$$

that is, $\pi^{i}\left(\tau_{1}(\gamma)\right)$ is an element in the nilpotent orbit in $\mathfrak{s p}\left(V^{i}\right)$ corresponding to $\left[2 r_{i}-1\right]^{+} \oplus\left[2 r_{i}-1\right]^{-}$. There is a self-dual set $S=\left\{v_{l}^{i}, w_{l}^{i} \mid l=1, \ldots, 2 r_{i}-2\right\}$ and two isotropic vectors $\omega_{0}^{i}$ and $\omega_{2 r_{i}-1}^{i}$, orthogonal to each other and orthogonal to $S$, such that $\gamma^{i}$ is of the form

$e_{1}^{i} \otimes v_{1}^{i}+e_{2}^{i} \otimes v_{2}^{i}+\cdots+e_{2 r^{i}-2}^{i} \otimes v_{2 r_{i}-2}^{i}+e_{2 r_{i}-1}^{i} \otimes \omega_{2 r_{i}-1}^{i}$

$$
+f_{1}^{i} \otimes \omega_{0}^{i}+f_{2}^{i} \otimes w_{1}^{i}+\cdots+f_{2 r_{i}-2}^{i} \otimes w_{2 r_{i}-3}^{i}+f_{2 r_{i}-1}^{i} \otimes w_{2 r_{i}-2}^{i} .
$$

Therefore, $\tau_{2}(\gamma)$ has a block of the form

$$
v_{1}^{i} \wedge \omega_{0}^{i}+v_{2}^{i} \wedge w_{1}^{i}+\cdots+v_{2 r_{i}-2}^{i} \wedge w_{2 r_{i}-3}^{i}+\omega_{2 r_{i}-1}^{i} \wedge w_{2 r_{i}-2}^{i}
$$

This block corresponds to

$$
\begin{cases}{\left[2 r_{i}\right]^{+} \oplus\left[2 r_{i}\right]^{-}} & \text {if both } \omega_{0}^{i} \text { and } \omega_{2 r_{i}-1}^{i} \text { are allowed to be nonzero; } \\ {\left[2 r_{i}-1\right]^{+} \oplus\left[2 r_{i}-1\right]^{-}} & \text {if only one of } \omega_{0}^{i} \text { and } \omega_{2 r_{i}-1}^{i} \text { is allowed to be nonzero; } \\ {\left[2 r_{i}-2\right]^{+} \oplus\left[2 r_{i}-2\right]^{-}} & \text {if both } \omega_{0}^{i} \text { and } \omega_{2 r_{i}-1}^{i} \text { have to be zero. }\end{cases}
$$

5.4. Example. Suppose that $2 n=8, p=4, q=4$ and $\boldsymbol{d}=[4]^{+} \oplus[2]^{-} \oplus[1]^{+} \oplus[1]^{-}$. Let $\gamma$ be an element in $V \otimes W$ such that $\tau_{1}(\gamma)$ is in the nilpotent orbit corresponding to $\boldsymbol{d}$. Now, $\boldsymbol{d}=\boldsymbol{d}^{1} \oplus \boldsymbol{d}^{2} \oplus \boldsymbol{d}^{3}$, where $\boldsymbol{d}^{1}:=[4]^{+}, \boldsymbol{d}^{2}:=[2]^{-}$and $\boldsymbol{d}^{3}:=[1]^{+} \oplus[1]^{-}$. We have the corresponding decomposition $V^{1} \oplus V^{2} \oplus V^{3}$ of $V$, where $V^{1}$ is 4dimensional, and $V^{2}$ and $V^{3}$ are 2-dimensional. Therefore, a nilpotent orbit $\mathrm{O}_{\boldsymbol{d}^{\prime}}$ in $\mathfrak{s o}(W)$ is in $\tau_{2}\left(\tau_{1}^{-1}\left(\mathrm{O}_{\boldsymbol{d}}\right)\right)$ if and only if

$$
\boldsymbol{d}^{\prime}=\boldsymbol{d}_{1}^{\prime} \oplus \boldsymbol{d}_{2}^{\prime} \oplus \boldsymbol{d}_{3}^{\prime} \oplus \frac{1}{2}\left(8-\left|\boldsymbol{d}_{1}^{\prime}\right|-\left|\boldsymbol{d}_{2}^{\prime}\right|-\left|\boldsymbol{d}_{2}^{\prime}\right|\right)\left([1]^{+} \oplus[1]^{-}\right)
$$

such that $\boldsymbol{d}_{1}^{\prime}$ is $[5]^{-}$or $[3]^{-}, \boldsymbol{d}_{2}^{\prime}$ is $[3]^{+}$or $[1]^{+}, \boldsymbol{d}_{3}^{\prime}$ is $[2]^{+} \oplus[2]^{-},[1]^{+} \oplus[1]^{-}$or $\varnothing$, and $\sum_{i=1}^{3}\left|\boldsymbol{d}_{i}^{\prime}\right| \leq 8$. The only possible combinations for $\boldsymbol{d}^{\prime}$ are

$$
\begin{array}{ll}
\boldsymbol{e}_{1}:=[5]^{-} \oplus[3]^{+}, & \boldsymbol{e}_{4}:=[3]^{-} \oplus[1]^{+} \oplus[2]^{+} \oplus[2]^{-}, \\
\boldsymbol{e}_{2}:=[5]^{-} \oplus[1]^{+} \oplus[1]^{+} \oplus[1]^{-}, & \boldsymbol{e}_{5}:=[3]^{-} \oplus[1]^{+} \oplus[1]^{+} \oplus[1]^{-} \oplus[1]^{+} \oplus[1]^{-}, \\
\boldsymbol{e}_{3}:=[3]^{-} \oplus[3]^{+} \oplus[1]^{+} \oplus[1]^{-} . &
\end{array}
$$

Hence,

$$
\tau_{2}\left(\tau_{1}^{-1}\left(\mathcal{O}_{\boldsymbol{d}}\right)\right)=\sigma_{\boldsymbol{e}_{1}} \cup \sigma_{\boldsymbol{e}_{2}} \cup \mathrm{O}_{\boldsymbol{e}_{3}} \cup \mathrm{O}_{\boldsymbol{e}_{4}} \cup \mathrm{O}_{\boldsymbol{e}_{5}}
$$




\section{The construction for real cases}

In this section, we assume that $F=\mathbb{R}$, that $V$ is a $2 n$-dimensional real symplectic space and that $W$ is an $m$-dimensional real quadratic space with signature $(p, q)$. Let $\tau_{1}$ and $\tau_{2}$ be the moment maps given in 3.4.

6.1. Our purpose is to define an orthogonal signed partition $\Phi(\lambda(\boldsymbol{d}, Q))$ for an admissible signed Young diagram $(\boldsymbol{d}, Q)$ (see 2.3), such that the following main theorem holds:

Theorem. Let $\mathrm{O}_{\boldsymbol{d}}$ be a nilpotent orbit in $\mathfrak{s p}(V)$ contained in the image of $\tau_{1}$. Then

$$
\tau_{2}\left(\tau_{1}^{-1}\left(\overline{\mathscr{O}}_{\boldsymbol{d}}\right)\right)=\bigcup_{(\boldsymbol{d}, Q)} \overline{\bar{O}_{\Phi(\lambda(\boldsymbol{d}, Q))}},
$$

where the union runs over all possible admissible signed Young diagrams of the signed partition $\boldsymbol{d}$. The union consists of at most two closures of nilpotent orbits.

The theorem will be proved in 7.8.

6.2. Let

$$
\boldsymbol{d}:=\bigoplus_{i=1}^{k} \alpha_{i}\left[a_{i}\right]^{+} \oplus \bigoplus_{j=1}^{l} \beta_{j}\left[b_{j}\right]^{-}
$$

be a symplectic signed partition of sized $2 n$, where $a_{1}>a_{2}>\cdots>a_{k}>0$ and $b_{1}>b_{2}>\cdots>b_{l}>0$. We can rewrite $\boldsymbol{d}$ as

$$
\boldsymbol{d}=\bigoplus_{i=1}^{k} \bigoplus_{j=1}^{\alpha_{i}}\left[a_{i}^{j}\right]^{+} \oplus \bigoplus_{i=1}^{l} \bigoplus_{j=1}^{\beta_{i}}\left[b_{i}^{j}\right]^{-}
$$

such that the numbers $a_{i}^{j}$ and $b_{i}^{j}$ satisfy the conditions

$$
\begin{aligned}
& a_{1}^{1}=\cdots=a_{1}^{\alpha_{1}}>a_{2}^{1}=\cdots=a_{2}^{\alpha_{2}}>\cdots>a_{k}^{1}=\cdots=a_{k}^{\alpha_{k}}, \\
& b_{1}^{1}=\cdots=b_{1}^{\beta_{1}}>b_{2}^{1}=\cdots=b_{2}^{\beta_{2}}>\cdots>b_{l}^{1}=\cdots=b_{l}^{\beta_{l}},
\end{aligned}
$$

where $a_{i}^{1}=\cdots=a_{i}^{\alpha_{i}}=a_{i}$ and $b_{j}^{1}=\cdots=b_{j}^{\beta_{j}}=b_{j}$ for each $i$ and $j$. To simplify the notation, we define an order on the set $\left\{\left[a_{x}^{y}\right]^{+} \mid x, y\right\}$, as follows. We say that $\left[a_{x_{1}}^{y_{1}}\right]^{+}>\left[a_{x_{2}}^{y_{2}}\right]^{+}$if either $x_{1}<x_{2}$, or $x_{1}=x_{2}$ and $y_{1}<y_{2}$. It is a total order on the set $\left\{\left[a_{x}^{y}\right]^{+} \mid x, y\right\}$; that is, we have the sequence

$$
\begin{aligned}
{\left[a_{1}^{1}\right]^{+}>\cdots>\left[a_{1}^{\alpha_{1}}\right]^{+} } & >\left[a_{2}^{1}\right]^{+}>\cdots>\left[a_{2}^{\alpha_{2}}\right]^{+}>\cdots \\
& >\left[a_{k-1}^{1}\right]^{+}>\cdots>\left[a_{k-1}^{\alpha_{k-1}}\right]^{+}>\left[a_{k}^{1}\right]^{+}>\cdots>\left[a_{k}^{\alpha_{k}}\right]^{+} .
\end{aligned}
$$

We define a total order on the set $\left\{\left[b_{x}^{y}\right]^{-} \mid x, y\right\}$ analogously. 
6.3. The ten cases. We want to define two signed partitions $\psi_{1}(\boldsymbol{d})$ and $\psi_{2}(\boldsymbol{d})$ from the symplectic signed partition $\boldsymbol{d}$. The definition depends on the signature $(p, q)$ of $W$. Without loss of generality we assume that $p \geq q$. Let $\alpha:=\sum_{i=1}^{k} \alpha_{i}$, $\beta:=\sum_{j=1}^{l} \beta_{j}, d_{1}:=\min \{p-n, \beta\}$ and $d_{2}:=\min \{q-n, \alpha\}$. We define $\psi_{1}(\boldsymbol{d})$ and $\psi_{2}(\boldsymbol{d})$ according to the following cases:

(i) Suppose $q \geq n$ and $\beta-\alpha \geq p-n$. We have $p \geq n$ and $\beta>\beta-\alpha-(p-n) \geq 0$. Therefore, there exists a unique number $v$ between 0 and $l$ such that $\sum_{i=v}^{l} \beta_{i}>$ $\beta-\alpha-(p-n) \geq \sum_{i=v+1}^{l} \beta_{i}$. Let $\beta^{\prime}:=\sum_{i=v}^{l} \beta_{i}-(\beta-\alpha-(p-n))$, and define

$$
\begin{aligned}
& \psi_{1}(\boldsymbol{d}):=\left(\bigoplus_{i=1}^{k} \bigoplus_{j=1}^{\alpha_{i}}\left[a_{i}^{j}\right]^{+}\right) \oplus\left(\bigoplus_{\left[b_{x}^{y}\right]^{-} \geq\left[b_{v}^{\beta^{\prime}}\right]^{-}}\left[b_{x}^{y}\right]^{-}\right) \oplus(p-n)[1]^{+}, \\
& \psi_{2}(\boldsymbol{d}):=\left(\bigoplus_{\left[b_{x}^{y}\right]^{-}<\left[b_{v}^{\beta^{\prime}}\right]^{-}}\left[b_{x}^{y}-1\right]^{+}\right) \oplus(\beta-\alpha+q-p)[1]^{-} .
\end{aligned}
$$

(ii) Suppose that $q \geq n$ and $p-n>\beta-\alpha \geq p-q$. It is clear that both $d_{1}$ and $p-n-d_{1}$ are nonnegative. Now, $\alpha-\beta+d_{1}$ is equal to $\alpha$ or $\alpha-\beta+p-n$, and hence $\alpha-\beta+d_{1}$ is also nonnegative. Now, $\beta-\alpha+q-n-d_{1} \geq p-n-d_{1}$. So, $\beta-\alpha+q-n-d_{1}$ is nonnegative. Define

$$
\begin{aligned}
& \psi_{1}(\boldsymbol{d}):=\boldsymbol{d} \oplus d_{1}[1]^{+} \oplus\left(\alpha-\beta+d_{1}\right)[1]^{-}, \\
& \psi_{2}(\boldsymbol{d}):=\left(p-n-d_{1}\right)[1]^{+} \oplus\left(\beta-\alpha+q-n-d_{1}\right)[1]^{-} .
\end{aligned}
$$

(iii) Suppose that $q \geq n$ and $p-q>\beta-\alpha \geq-(q-n)$. Clearly both $d_{2}$ and $q-n-d_{2}$ are nonnegative. Now, $\beta-\alpha+d_{2}$ is equal to $\beta$ or $\beta-\alpha+(q-n)$. Hence, $\beta-\alpha+d_{2}$ is nonnegative. Next, $\alpha-\beta+p-n-d_{2}>q-n-d_{2}$. So, $\alpha-\beta+p-n-d_{2}$ is also nonnegative. Define

$$
\begin{aligned}
& \psi_{1}(\boldsymbol{d}):=\boldsymbol{d} \oplus\left(\beta-\alpha+d_{2}\right)[1]^{+} \oplus d_{2}[1]^{-} \\
& \psi_{2}(\boldsymbol{d}):=\left(\alpha-\beta+p-n-d_{2}\right)[1]^{+} \oplus\left(q-n-d_{2}\right)[1]^{-} .
\end{aligned}
$$

(iv) Suppose that $q \geq n$ and $-(q-n)>\beta-\alpha$. Since $\alpha \geq \alpha-\beta-(q-n)>0$, there is a unique number $\mu$ between 0 and $k$ such that $\sum_{i=\mu}^{k} \alpha_{i}>\alpha-\beta-(q-n) \geq$ $\sum_{i=\mu+1}^{k} \alpha_{i}$. Let $\alpha^{\prime}:=\sum_{i=\mu}^{k} \alpha_{i}-(\alpha-\beta-(q-n))$. Define

$$
\begin{aligned}
& \psi_{1}(\boldsymbol{d}):=\left(\bigoplus_{\left[a_{x}^{y}\right]^{+} \geq\left[a_{\mu}^{\alpha^{\prime}}\right]^{+}}\left[a_{x}^{y}\right]^{+}\right) \oplus\left(\bigoplus_{i=1}^{l} \bigoplus_{j=1}^{\beta_{i}}\left[b_{i}^{j}\right]^{-}\right) \oplus(q-n)[1]^{-}, \\
& \psi_{2}(\boldsymbol{d}):=\left(\bigoplus_{\left[a_{x}^{y}\right]^{+}<\left[a_{\mu}^{\alpha^{\prime}}\right]^{+}}\left[a_{x}^{y}-1\right]^{-}\right) \oplus(\alpha-\beta+p-q)[1]^{+} .
\end{aligned}
$$


(v) Suppose that $p \geq n>q \geq n-\beta$ and $\beta-\alpha \geq p-q$. We have $\beta \geq \beta-\alpha-(p-n) \geq$ $n-q>0$. Let $v$ be the number such that

$$
\sum_{i=v}^{l} \beta_{i}>\beta-\alpha-(p-n) \geq \sum_{i=v+1}^{l} \beta_{i},
$$

and let $\beta^{\prime}:=\sum_{i=v}^{l} \beta_{i}-(\beta-\alpha-(p-n))$. Define

$$
\begin{aligned}
& \psi_{1}(\boldsymbol{d}):=\left(\bigoplus_{i=1}^{k} \bigoplus_{j=1}^{\alpha_{i}}\left[a_{i}^{j}\right]^{+}\right) \oplus\left(\bigoplus_{\left[b_{x}^{y}\right]^{-} \geq\left[b_{v}^{\beta^{\prime}}\right]^{-}}\left[b_{x}^{y}\right]^{-}\right) \oplus(p-n)[1]^{+}, \\
& \psi_{2}(\boldsymbol{d}):=\left(\bigoplus_{\left[b_{x}^{y}\right]^{-}<\left[b_{v}^{\beta^{\prime}}\right]^{-}}\left[b_{x}^{y}-1\right]^{+}\right) \oplus(\beta-\alpha+q-p)[1]^{-} .
\end{aligned}
$$

(vi) Suppose that $p \geq n>q \geq n-\beta$ and $p-q>\beta-\alpha \geq n-q$. Now, both $\beta-\alpha+q-n$ and $p-q-\beta+\alpha$ are nonnegative. Since $\beta \geq n-q>0$, there is a unique number $v^{\prime}$ such that $\sum_{i=v^{\prime}}^{l} \beta_{i}>n-q \geq \sum_{i=v^{\prime}+1}^{l} \beta_{i}$. Let $\beta^{\prime \prime}:=\sum_{i=v^{\prime}}^{l} \beta_{i}-(n-q)$. Define

$$
\begin{aligned}
& \psi_{1}(\boldsymbol{d}):=\left(\bigoplus_{i=1}^{k} \bigoplus_{j=1}^{\alpha_{i}}\left[a_{i}^{j}\right]^{+}\right) \oplus\left(\bigoplus_{\left[b_{x}^{y}\right]^{-} \geq\left[b_{v^{\prime}}^{\beta^{\prime \prime}}\right]^{-}}\left[b_{x}^{y}\right]^{-}\right) \oplus(\beta-\alpha+q-n)[1]^{+}, \\
& \psi_{2}(\boldsymbol{d}):=\left(\bigoplus_{\left[b_{x}^{y}\right]^{-}<\left[b_{v^{\prime}}^{\beta^{\prime \prime}}\right]^{-}}\left[b_{x}^{y}-1\right]^{+}\right) \oplus(\alpha-\beta+p-q)[1]^{+} .
\end{aligned}
$$

(vii) Suppose that $p \geq n>q \geq n-\beta$ and $n-q>\beta-\alpha$. Hence, $\alpha \geq \alpha-\beta-q+n>0$ and $\beta \geq n-q>0$. Let $\mu$ and $\nu^{\prime}$ be the numbers such that

$\sum_{i=\mu}^{k} \alpha_{i}>\alpha-\beta-(q-n) \geq \sum_{i=\mu+1}^{k} \alpha_{i} \quad$ and $\quad \sum_{i=\nu^{\prime}}^{l} \beta_{i}>n-q \geq \sum_{i=\nu^{\prime}+1}^{l} \beta_{i}$.

Let $\alpha^{\prime}:=\sum_{i=1}^{\mu} \alpha_{i}-(\alpha-\beta-(q-n))$ and $\beta^{\prime \prime}:=\sum_{i=1}^{v^{\prime}} \beta_{i}-(n-q)$. Now, $\alpha-\beta+p-q \geq \alpha-\beta+n-q \geq 0$. Define

$$
\begin{aligned}
& \psi_{1}(\boldsymbol{d}):=\left(\bigoplus_{\left[a_{x}^{y}\right]^{+} \geq\left[a_{\mu}^{\alpha^{\prime}}\right]^{+}}\left[a_{x}^{y}\right]^{+}\right) \oplus\left(\bigoplus_{\left[b_{x}^{y}\right]^{-} \geq\left[b_{v^{\prime}}^{\beta^{\prime \prime}}\right]^{-}}\left[b_{x}^{y}\right]^{-}\right), \\
& \psi_{2}(\boldsymbol{d}):=\left(\bigoplus_{\left[a_{x}^{y}\right]^{+}<\left[a_{\mu}^{\alpha^{\prime}}\right]^{+}}\left[a_{x}^{y}-1\right]^{-}\right) \oplus\left(\bigoplus_{\left[b_{x}^{y}\right]^{-}<\left[b_{v^{\prime}}^{\beta^{\prime \prime}}\right]^{-}}\left[b_{x}^{y}-1\right]^{+}\right) \oplus(\alpha-\beta+p-q)[1]^{+} .
\end{aligned}
$$

(viii) Suppose that $n>p \geq n-\alpha, n>q \geq n-\beta$ and $\beta-\alpha \geq p-q$. Hence, $\alpha \geq n-p>0$ and $\beta \geq \beta-\alpha+n-p>0$. Let $\mu^{\prime}$ and $v$ be the numbers such that

$\sum_{i=\mu^{\prime}}^{k} \alpha_{i}>n-p \geq \sum_{i=\mu^{\prime}+1}^{k} \alpha_{i} \quad$ and $\quad \sum_{i=v}^{l} \beta_{i}>\beta-\alpha-(p-n) \geq \sum_{i=v+1}^{l} \beta_{i}$. 
Let $\alpha^{\prime \prime}:=\sum_{i=1}^{\mu} \alpha_{i}-(n-p)$ and $\beta^{\prime}:=\sum_{i=v}^{l} \beta_{i}-(\beta-\alpha-(p-n))$. Define $\psi_{1}(\boldsymbol{d}):=\left(\bigoplus_{\left[a_{x}^{y}\right]^{+}>\left[a_{\mu^{\prime}}^{\alpha^{\prime \prime}}\right]^{+}}\left[a_{x}^{y}\right]^{+}\right) \oplus\left(\bigoplus_{\left[b_{x}^{y}\right]^{-}>\left[b_{v}^{\beta^{\prime}}\right]^{-}}\left[b_{x}^{y}\right]^{-}\right)$, $\psi_{2}(\boldsymbol{d}):=\left(\bigoplus_{\left[a_{x}^{y}\right]^{+} \leq\left[a_{\mu^{\prime}}^{\left.\alpha^{\prime \prime}\right]^{+}}\right.}\left[a_{x}^{y}-1\right]^{-}\right) \oplus\left(\bigoplus_{\left[b_{x}^{y}\right]^{-} \leq\left[b_{v}^{\beta^{\prime}}\right]^{-}}\left[b_{x}^{y}-1\right]^{+}\right) \oplus(\beta-\alpha+q-p)[1]^{-}$.

(ix) Suppose that $n>p \geq n-\alpha, n>q \geq n-\beta$ and $p-q>\beta-\alpha$. Hence, $\alpha \geq \alpha-\beta+n-q>0$ and $\beta \geq n-q>0$. Let $\mu$ and $v^{\prime}$ be the numbers such that $\sum_{i=\mu}^{k} \alpha_{i}>\alpha-\beta-(q-n) \geq \sum_{i=\mu+1}^{k} \alpha_{i}$ and $\sum_{i=v^{\prime}}^{l} \beta_{i}>n-q \geq \sum_{i=v^{\prime}+1}^{l} \beta_{i}$. Let $\alpha^{\prime}:=\sum_{i=\mu}^{k} \alpha_{i}-(\alpha-\beta-(q-n))$ and $\beta^{\prime \prime}:=\sum_{i=\nu}^{l} \beta_{i}-(n-q)$. Define $\psi_{1}(\boldsymbol{d}):=\left(\bigoplus_{\left[a_{x}^{y}\right]^{+} \geq\left[a_{\mu}^{\alpha^{\prime}}\right]^{+}}\left[a_{x}^{y}\right]^{+}\right) \oplus\left(\bigoplus_{\left[b_{x}^{y}\right]^{-} \geq\left[b_{v^{\prime}}^{\beta^{\prime \prime}}\right]^{-}}\left[b_{x}^{y}\right]^{-}\right)$, $\psi_{2}(\boldsymbol{d}):=\left(\bigoplus_{\left[a_{x}^{y}\right]^{+}<\left[a_{\mu}^{\left.\alpha^{\prime}\right]^{+}}\right.}\left[a_{x}^{y}-1\right]^{-}\right) \oplus\left(\bigoplus_{\left[b_{x}^{y}\right]^{-}<\left[b_{v^{\prime}}^{\beta^{\prime \prime}}\right]^{-}}\left[b_{x}^{y}-1\right]^{+}\right) \oplus(\alpha-\beta+p-q)[1]^{+}$.

(x) Suppose that $p<n-\alpha$, or $q<n-\beta$. In this case we define

$$
\psi_{1}(\boldsymbol{d})=\psi_{2}(\boldsymbol{d}):=\varnothing \text {. }
$$

6.4. Lemma. Let $\psi_{1}(\boldsymbol{d})$ be defined as in 6.3. If we write

$$
\psi_{1}(\boldsymbol{d}):=\bigoplus_{i=1}^{k^{\prime}} \bigoplus_{j=1}^{\alpha_{i}^{\prime}}\left[a_{i}^{\prime j}\right]^{+} \oplus \bigoplus_{i=1}^{l^{\prime}} \bigoplus_{j=1}^{\beta_{i}^{\prime}}\left[b_{i}^{j^{j}}\right]^{-},
$$

then $\sum_{i=1}^{k^{\prime}} \alpha_{i}^{\prime}=\sum_{i=1}^{l^{\prime}} \beta_{i}^{\prime}$; that is, the number of positive rows of $\psi_{1}(\boldsymbol{d})$ is equal to the number of negative rows.

Proof. For case (i) in 6.3, we have

$\sum_{i=1}^{k^{\prime}} \alpha_{i}^{\prime}=\sum_{i=1}^{k} \alpha_{i}+p-n=\alpha+p-n$,

$\sum_{i=1}^{l^{\prime}} \beta_{i}^{\prime}=\sum_{i=1}^{v} \beta_{i}+\beta^{\prime}=\sum_{i=1}^{v} \beta_{i}+\sum_{i=1}^{v} \beta_{i}-(\beta-\alpha-(p-n))=\alpha-(p-n)$.

Hence, $\sum_{i=1}^{k^{\prime}} \alpha_{i}^{\prime}=\sum_{i=1}^{l^{\prime}} \beta_{i}^{\prime}$ for this case.

Next, we consider case (ii). We have

$$
\begin{aligned}
& \sum_{i=1}^{k^{\prime}} \alpha_{i}^{\prime}=\sum_{i=1}^{k} \alpha_{i}+d_{1}=\alpha+d_{1} \\
& \sum_{i=1}^{l^{\prime}} \beta_{i}^{\prime}=\sum_{i=1}^{l} \beta_{i}+d_{1}-(\beta-\alpha)=\alpha+d_{1} .
\end{aligned}
$$

Hence, $\sum_{i=1}^{k^{\prime}} \alpha_{i}^{\prime}=\sum_{i=1}^{l^{\prime}} \beta_{i}^{\prime}$. The proofs for the other cases are similar, and we skip them.

From this lemma we know that the number of rows of $\psi_{1}(\boldsymbol{d})$ is even. 
6.5. Lemma. Let $\left[r_{x_{1}}^{y_{1}}\right]^{*}<\left[r_{x_{2}}^{y_{2}}\right]^{*}$ be the two median elements with respect to an order on the set of rows of $\psi_{1}(\boldsymbol{d})$. If $\left[r_{x}^{y-1}\right]^{*} \oplus\left[r_{x}^{y}\right]^{*}$ is a double odd block of $\boldsymbol{d}$ such that $\left[r_{x}^{y}\right]^{*}$ is in $\psi_{1}(\boldsymbol{d})$ but $\left[r_{x}^{y-1}\right]^{*}$ is not in $\psi_{1}(\boldsymbol{d})$, then $\left[r_{x}^{y}\right]^{*} \leq\left[r_{x_{1}}^{y_{1}}\right]^{*}$ with respect to the same order.

Proof. For case (i) in 6.3, $\left[r_{x}^{y}\right]^{*}$ is $\left[r_{x}^{y}\right]^{+}$; that is, it ends with $\boxplus$. If $\left[r_{z}^{w}\right]^{*}<\left[r_{x}^{y}\right]^{+}$, then $\left[r_{z}^{w}\right]^{*}$ also ends with $\boxplus$. The lemma follows from 6.4, because no more than half the number of rows end with $\boxplus$. For cases (ii) and (iii), the lemma is true because no such $\left[r_{x}^{y}\right]^{*}$ exist. For case (iv), $\left[r_{x}^{y}\right]^{*}$ is $\left[r_{x}^{y}\right]^{-}$; that is, it ends with $\boxminus$. If $\left[r_{z}^{w}\right]^{*}<\left[r_{x}^{y}\right]^{-}$, then $\left[r_{z}^{w}\right]^{*}$ also ends with $\boxminus$. This lemma holds again, by 6.4 . The proofs for the other cases are similar, and we omit them.

6.6. Minimal and maximal elements. We set $\varphi(d):=[0]$ for case $(x)$ in 6.3 , and

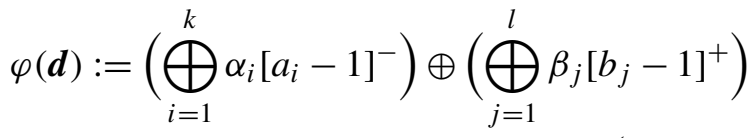

$$
\oplus(\alpha+p-n)[1]^{+} \oplus(\beta+q-n)[1]^{-}
$$

for the other cases.

Suppose that $\psi_{1}(\boldsymbol{d}) \neq[0]$. Let $\left(\psi_{1}(\boldsymbol{d}), Q\right)$ be a signed Young diagram of $\psi_{1}(\boldsymbol{d})$. Then, as in Equation (4-5), we can define the element $\Phi^{*}\left(\psi_{1}(\boldsymbol{d}), Q\right)$ as follows: Assume that $\psi_{1}(\boldsymbol{d})$ is written as $\bigoplus_{i=1}^{\delta} \bigoplus_{j=1}^{\rho_{i}}\left[f_{i}^{j}\right]^{*}$. Let $s_{x}^{y}$ be defined as in 4.4. If $s_{x}^{y}=f_{x}^{y}$, let the sign of $\left[s_{x}^{y}\right]^{*}$ be the same as that of $\left[f_{x}^{y}\right]^{*}$; if $s_{x}^{y} \neq f_{x}^{y}$, let the sign of $\left[s_{x}^{y}\right]^{*}$ be opposite to the sign of $\left[f_{x}^{y}\right]^{*}$. Finally, define

$$
\begin{aligned}
\Phi^{*}\left(\psi_{1}(\boldsymbol{d}), Q\right) & :=\bigoplus_{i=1}^{\delta} \bigoplus_{j=1}^{\rho_{i}}\left[s_{i}^{j}\right]^{*} \\
\Phi(\boldsymbol{d}, Q) & :=\Phi^{*}\left(\psi_{1}(\boldsymbol{d}), Q\right) \oplus \psi_{2}(\boldsymbol{d}) .
\end{aligned}
$$

Proposition. If $\Phi(\boldsymbol{d}, Q)$ and $\varphi(\boldsymbol{d})$ are not [0], then they are signed orthogonal partitions of signature $(p, q)$.

Proof. From Equation (6-4), it is clear that the signature of $\varphi(\boldsymbol{d})$ is $(p, q)$. We know that $\boldsymbol{d}$ is a finite sum of simple even blocks or double odd blocks. Hence, it is clear that $\varphi(\boldsymbol{d})$ is a finite sum of simple odd blocks or double even blocks. Therefore, $\varphi(\boldsymbol{d})$ is an orthogonal signed partition.

Next, we consider $\Phi(\boldsymbol{d}, Q)$. The meaning of 6.5 is that the image of two rows of a double block still have the same length after applying $\Phi^{*}$. Hence, from its definition, it is not difficult to see that $\Phi(\boldsymbol{d}, Q)$ is an orthogonal signed partition. If we check the definitions of $\psi_{1}(\boldsymbol{d})$ and $\psi_{2}(\boldsymbol{d})$, we see that the signature of $\psi_{1}(\boldsymbol{d}) \oplus \psi_{2}(\boldsymbol{d})$ is $(p, q)$. Clearly, the signature of $\psi_{1}(\boldsymbol{d})$ is the same as the signature of $\Phi^{*}\left(\psi_{1}(\boldsymbol{d}), Q\right)$ for any order $Q$. Hence, $\Phi(\boldsymbol{d}, Q)$ has signature $(p, q)$. 
Let $\mathcal{O}_{\boldsymbol{d}}$ be the nilpotent orbit in $\mathfrak{s p}(V)$ corresponding to $\boldsymbol{d}$. From the previous proposition, we know that each of $\varphi(\boldsymbol{d})$ and $\Phi(\boldsymbol{d}, Q)$ correspond to a nilpotent orbit in $\mathfrak{s o}(W)$. We define

$$
\varphi\left(\mathscr{O}_{\boldsymbol{d}}\right):=\mathscr{O}_{\varphi(\boldsymbol{d})} \quad \text { and } \quad \Phi\left(\mathscr{O}_{\boldsymbol{d}}\right):=\bigcup_{Q} \mathcal{O}_{\Phi(\boldsymbol{d}, Q)},
$$

where the union $\bigcup_{Q} \mathcal{O}_{\Phi(d, Q)}$ is running over all possible signed Young diagrams $\left(\psi_{1}(\boldsymbol{d}), Q\right)$ of the signed partition $\psi_{1}(\boldsymbol{d})$. Of course, it is a finite union.

6.7. Example. Suppose that $2 n=36, p=21, q=19$ and $\boldsymbol{d}=2[4]^{+} \oplus[3]^{+} \oplus$ $5[4]^{-} \oplus[3]^{-} \oplus[2]^{-}$. Now, $p-q=2, \alpha_{1}=2, \alpha_{2}=1, \alpha=2+1=3, \beta_{1}=5, \beta_{2}=1$, $\beta_{3}=1, \beta=5+1+1=7, d_{1}=\min \{21-18,7\}=3, d_{2}=\min \{19-18,3\}=1$ and $\beta-\alpha=4$. So, we are in case (i) of 6.3. Now, $\beta_{2}+\beta_{3}>\beta-\alpha-(p-n) \geq \beta_{3}$. Then, $v=2$ and $\beta^{\prime}=(1+1)-(7-3-(21-18))=1$. Therefore, $\psi_{1}(\boldsymbol{d})=$ $2[4]^{+} \oplus[3]^{+} \oplus 5[4]^{-} \oplus[3]^{-} \oplus 3[1]^{+}$and $\psi_{2}(\boldsymbol{d})=[1]^{+} \oplus 2[1]^{-}$. Note that the number of positive blocks in $\psi_{1}(\boldsymbol{d})$ is equal to the number of negative blocks. It is not difficult to see that there are 42 different signed Young diagrams $\left(\psi_{1}(\boldsymbol{d}), Q\right)$ of the signed partition $\psi_{1}(\boldsymbol{d})$. However, they only produce two different $\Phi^{*}\left(\psi_{1}(\boldsymbol{d}), Q\right)$, which are

$$
\begin{aligned}
& \Phi^{*}\left(\psi_{1}(\boldsymbol{d}), Q_{1}\right)=5[5]^{+} \oplus[2]^{+} \oplus[5]^{-} \oplus[3]^{-} \oplus[2]^{-}, \\
& \Phi^{*}\left(\psi_{1}(\boldsymbol{d}), Q_{2}\right)=4[5]^{+} \oplus[3]^{+} \oplus[2]^{+} \oplus 2[5]^{-} \oplus[2]^{-} .
\end{aligned}
$$

Therefore, $\Phi\left(\mathscr{O}_{\boldsymbol{d}}\right)=\mathscr{O}_{\boldsymbol{e}_{1}} \cup \mathscr{O}_{\boldsymbol{e}_{2}}$ and $\varphi\left(\mathscr{O}_{\boldsymbol{d}}\right)=\mathscr{O}_{\boldsymbol{f}}$, where

$$
\begin{aligned}
\boldsymbol{e}_{1} & :=5[5]^{+} \oplus[2]^{+} \oplus[1]^{+} \oplus[5]^{-} \oplus[3]^{-} \oplus[2]^{-} \oplus 2[1]^{-}, \\
\boldsymbol{e}_{2} & :=4[5]^{+} \oplus[3]^{+} \oplus[2]^{+} \oplus[1]^{+} \oplus 2[5]^{-} \oplus[2]^{-} \oplus 2[1]^{-}, \\
\boldsymbol{f} & :=5[3]^{+} \oplus[2]^{+} \oplus 7[1]^{+} \oplus 2[3]^{-} \oplus[2]^{-} \oplus 8[1]^{-} .
\end{aligned}
$$

6.8. Admissible signed Young diagrams. Let $\boldsymbol{d}$ be a symplectic signed partition, as in 6.2. Rewrite $\boldsymbol{d}$ as

$$
\boldsymbol{d}=\bigoplus_{i=1}^{s} \bigoplus_{j=1}^{\gamma_{i}}\left[r_{i}^{j}\right]^{\bullet},
$$

where each $\bullet$ is plus or minus, as usual, and these $r_{i}^{j}$ satisfy the condition $r_{1}^{1}=$ $\cdots=r_{1}^{\gamma_{1}}>r_{2}^{1}=\cdots=r_{2}^{\gamma_{2}}>\cdots>r_{s}^{1}=\cdots=r_{s}^{\gamma_{s}}$. Let $r_{x}$ be the size of a row $\left[r_{x}^{y}\right]$. We define a special kind of signed Young diagrams of $\boldsymbol{d}$ called admissible, as follows: The definition depends on the signature $(p, q)$ of $W$. Recall that we assume that $p \geq q$ and, in a Young diagram, a row of longer length is always above a shorter row.

(i) Among the rows of same length, if the length $r_{x}$ is even, we put a row $\left[r_{x}^{y}\right]^{*}$ with sign such that the difference of the negative rows and the positive rows that are no lower than the row $\left[r_{x}^{y}\right]^{*}$ is as close to $q-p$ as possible. 
(ii) If $r_{x}$ is odd, then the number of positive rows of length $r_{x}$ is equal to the number of negative rows of the same length. Let the signs of these $\left[r_{x}^{y}\right]^{*}$ appear alternatively, in the order starting from the sign such that the difference of negative rows and positive rows that are no lower than the row $\left[r_{x}^{y}\right]^{*}$ is as close to $p-q$ as possible.

If $(\boldsymbol{d}, Q)$ is an admissible signed Young diagram of $\boldsymbol{d}$, then $Q$ is called an admissible order on the set of rows in the signed partition $\boldsymbol{d}$.

6.9. Example. Suppose that $2 n=26, \quad p=14, q=13$ and $\boldsymbol{d}=2[4]^{+} \oplus 3[4]^{-} \oplus$ $[3]^{+} \oplus[3]^{-}$. The rows of $\boldsymbol{d}$ have two lengths, namely 4 and 3. So, the top five rows are of size 4 , and the next two rows are of size 3. Since now $p-q=1$, the first row of an admissible signed Young diagram of $\boldsymbol{d}$ must be negative. The second row is allowed to be positive or negative. If the second row is positive, then the third row has to be negative. If the second row is negative, then the third row is forced to be positive, and so on. Therefore, we have the following eight possible admissible signed Young diagrams associated to the signed partition $\boldsymbol{d}$ :

\begin{tabular}{|c|c|c|}
\hline 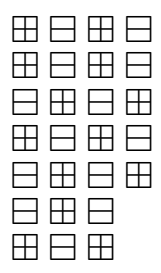 & 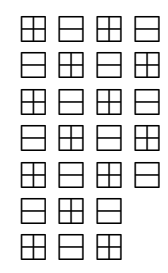 & 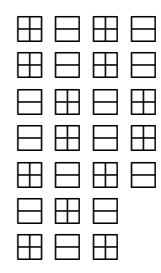 \\
\hline 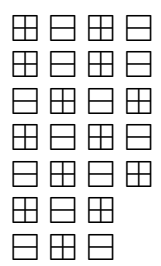 & 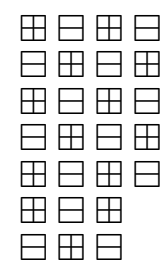 & 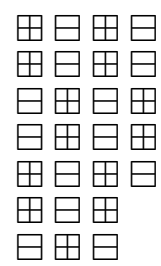 \\
\hline
\end{tabular}

6.10. Let $(\boldsymbol{d}, Q)$ be an admissible signed Young diagram of a symplectic signed partition $\boldsymbol{d}$ of size $2 n$. Recall that $Q$ is an order on the set $\left\{\left[r_{x}^{y}\right]^{*}\right\}$. Let $\left[r_{x_{0}}^{y_{0}}\right]^{*}$ be the smallest element under the order $Q$, subject to the condition that $v \leq p^{\prime}+p-n$ and $u \leq q^{\prime}+q-n$, where

$$
\left(p^{\prime}, q^{\prime}\right):=\operatorname{sgn}\left(\bigoplus_{\left[r_{x}^{y}\right]^{*} \leq\left[r_{x_{0}}^{y_{0}}\right]^{*}}\left[r_{x}^{y}\right]^{*}\right),
$$

$u$ is the cardinality of the set $\left\{\left[r_{x}^{y}\right]^{+} \mid\left[r_{x}^{y}\right]^{+}>\left[r_{x_{0}}^{y_{0}}\right]^{*}\right\}$ and $v$ is the cardinality of the set $\left\{\left[r_{x}^{y}\right]^{-} \mid\left[r_{x}^{y}\right]^{-}>\left[r_{x_{0}}^{y_{0}}\right]^{*}\right\}$. Set

$$
\sigma:=\min \left\{p^{\prime}+p-n-v, q^{\prime}+q-n-u\right\} .
$$


Let $[2 \sigma]^{*}$ be a simple signed partition, with the same sign as the sign of the row next to $\left[r_{x_{0}}^{y_{0}}\right]^{*}$. We define $\lambda(\boldsymbol{d}, Q)$ according to the following three cases:

(i) If $r_{x_{0}}^{y_{0}}$ is odd, $y_{0}$ is even, and $\left[r_{x_{0}}^{y_{0}}\right]^{*}$ is a positive row, then we define $\lambda(\boldsymbol{d}, Q):=\bigoplus_{\left[r_{x}^{y}\right]^{*}>\left[r_{x_{0}}^{y_{0}-1}\right]^{*}}\left[r_{x}^{y}\right]^{*} \oplus\left[r_{x_{0}}^{y_{0}-1}-1\right]^{-} \oplus[2 \sigma]^{*} \oplus\left(p^{\prime}-\sigma\right)[1]^{+} \oplus\left(q^{\prime}-\sigma+1\right)[1]^{-}$.

(ii) If $r_{x_{0}}^{y_{0}}$ is odd, $y_{0}$ is even, and $\left[r_{x_{0}}^{y_{0}}\right]^{*}$ is a negative row, then we define $\lambda(\boldsymbol{d}, Q):=\bigoplus_{\left[r_{x}^{y}\right]^{*}>\left[r_{x_{0}}-1\right.}\left[r_{x}^{y}\right]^{*} \oplus\left[r_{x_{0}}^{y_{0}-1}-1\right]^{+} \oplus[2 \sigma]^{*} \oplus\left(p^{\prime}-\sigma+1\right)[1]^{+} \oplus\left(q^{\prime}-\sigma\right)[1]^{-}$.

(iii) In the remaining cases, set

$$
\lambda(\boldsymbol{d}, Q):=\bigoplus_{\left[r_{x}^{y}\right]^{*}>\left[r_{x_{0}}^{y_{0}}\right]^{*}}\left[r_{x}^{y}\right]^{*} \oplus[2 \sigma]^{*} \oplus\left(p^{\prime}-\sigma\right)[1]^{+} \oplus\left(q^{\prime}-\sigma\right)[1]^{-} .
$$

Clearly, $\lambda(\boldsymbol{d}, Q)$ is a signed partition of signature $(n, n)$. Moreover, we can check that $p^{\prime}+1=q^{\prime}$ for case (i), $p^{\prime}=q^{\prime}+1$ for case (ii), and $p^{\prime}=q^{\prime}$ for case (iii). Hence, $\lambda(\boldsymbol{d}, Q)$ is a symplectic signed partition.

6.11. Example. Suppose $2 n=22, \boldsymbol{d}=[6]^{+} \oplus[6]^{-} \oplus[5]^{+} \oplus[5]^{-}$, and $p=q=11$. There are four admissible orders on the set of rows of $\boldsymbol{d}$, namely

$$
\begin{array}{ll}
Q_{1}: & {[6]^{+}>[6]^{-}>[5]^{+}>[5]^{-},} \\
Q_{2}: & {[6]^{-}>[6]^{+}>[5]^{+}>[5]^{-},} \\
Q_{3}: & {[6]^{+}>[6]^{-}>[5]^{-}>[5]^{+},} \\
Q_{4}: & {[6]^{-}>[6]^{+}>[5]^{-}>[5]^{+} .}
\end{array}
$$

First, we consider $Q_{1}$. Under the admissible order $Q_{1}$, it is easy to check that $\left[r_{x_{0}}^{y_{0}}\right]^{*}=[5]^{-}$. Then, $\left(p^{\prime}, q^{\prime}\right)=(2,3), u=2$ and $v=1$. Hence,

$$
\sigma=\min \{2+11-11-1,3+11-11-2\}=1 .
$$

Now, $r_{x_{0}}^{y_{0}}=5, y_{0}$ is 2 , and $\left[r_{x_{0}}^{y_{0}}\right]^{*}$ is a negative row. So, we are in case (ii) of 6.10 , and

$$
\lambda\left(\boldsymbol{d}, Q_{1}\right)=[6]^{+} \oplus[6]^{-} \oplus[4]^{+} \oplus[2]^{-} \oplus 2[1]^{+} \oplus 2[1]^{-} .
$$

For $Q_{2}$, we have $\left[r_{x_{0}}^{y_{0}}\right]^{*}=[5]^{-}$. By the same argument, we have

$$
\lambda\left(\boldsymbol{d}, Q_{2}\right)=[6]^{+} \oplus[6]^{-} \oplus[4]^{+} \oplus[2]^{-} \oplus 2[1]^{+} \oplus 2[1]^{-} .
$$

For $Q_{3}$, we have $\left[r_{x_{0}}^{y_{0}}\right]^{*}=[5]^{+}$. Then, $\left(p^{\prime}, q^{\prime}\right)=(3,2), u=1$ and $v=2$. Hence, $\sigma=\min \{3+11-11-2,2+11-11-1\}=1$. Now, $r_{x_{0}}^{y_{0}}=5, y_{0}$ is 2 and $\left[r_{x_{0}}^{y_{0}}\right]^{*}$ is a positive row. So, we are in case (i) of 6.10, and

$$
\lambda\left(\boldsymbol{d}, Q_{3}\right)=[6]^{+} \oplus[6]^{-} \oplus[4]^{-} \oplus[2]^{+} \oplus 2[1]^{+} \oplus 2[1]^{-} .
$$


For $Q_{4}$, we have $\left[r_{x_{0}}^{y_{0}}\right]^{*}=[5]^{+}$. By the same argument as for $Q_{3}$, we have

$$
\lambda\left(\boldsymbol{d}, Q_{4}\right)=[6]^{+} \oplus[6]^{-} \oplus[4]^{-} \oplus[2]^{+} \oplus 2[1]^{+} \oplus 2[1]^{-} .
$$

Note that $\lambda\left(\boldsymbol{d}, Q_{1}\right)=\lambda\left(\boldsymbol{d}, Q_{2}\right) \neq \lambda\left(\boldsymbol{d}, Q_{3}\right)=\lambda\left(\boldsymbol{d}, Q_{4}\right)$.

6.12. Lemma. For all possible admissible signed Young diagrams $(\boldsymbol{d}, Q)$ of a signed partition $\boldsymbol{d}$, there are at most two different signed partitions $\lambda(\boldsymbol{d}, Q)$.

Proof. Let $[a]^{*}$ be a row of the signed partition $\boldsymbol{d}$. Suppose that $[a]^{*}$ is $\left[r_{x_{0}}^{y_{0}}\right]^{*}$, under some admissible order $Q$. If $[a]^{*}$ is a row in a double odd block with partner $[b]^{*}$, then it might be that there is another admissible order $Q^{\prime}$ such that $\left[r_{x_{0}}^{y_{0}}\right]^{*}$ is $[b]^{*}$. If $[a]^{*}$ is a row of even length, it is also possible that there is another admissible order $Q^{\prime}$ such that $\left[r_{x_{0}}^{y_{0}}\right]^{*}$ is a row of the same length, but opposite sign. From the construction, we know that these two situations are the only chances to obtain different signed partitions $\lambda(\boldsymbol{d}, Q), \lambda\left(\boldsymbol{d}, Q^{\prime}\right)$.

6.13. Lemma. If $(\boldsymbol{d}, Q)$ is an admissible signed Young diagram of a signed partition $\boldsymbol{d}$, then $\lambda(\boldsymbol{d}, Q) \leq \boldsymbol{d}$ and $\Phi\left(\mathcal{O}_{\lambda(\boldsymbol{d}, Q)}\right)$ is a single nilpotent orbit.

Proof. The first part is obvious from the definition of $\lambda(\boldsymbol{d}, Q)$ in 6.10 .

We prove the second part. Let $Q_{1}$ and $Q_{2}$ be two different admissible orders of the signed partition $\psi_{1}(\lambda(\boldsymbol{d}, Q))$. From the construction of $\lambda(\boldsymbol{d}, Q)$ and definition of $\Phi$, it is not difficult to see that $\Phi^{*}\left(\psi_{1}(\lambda(\boldsymbol{d}), Q), Q_{1}\right)=\Phi^{*}\left(\psi_{1}(\lambda(\boldsymbol{d}), Q), Q_{2}\right)$. Hence,

$$
\Phi\left(\lambda(\boldsymbol{d}, Q), Q_{1}\right)=\Phi\left(\lambda(\boldsymbol{d}, Q), Q_{2}\right) .
$$

Therefore, $\Phi\left(\mathcal{O}_{\lambda(\boldsymbol{d}, Q)}\right)$ is a single nilpotent orbit.

Because the signed partition $\Phi\left(\lambda(\boldsymbol{d}, Q), Q^{\prime}\right)$ is independent of the admissible order $Q^{\prime}$ of $\psi_{1}(\lambda(\boldsymbol{d}, Q))$, we will write $\Phi(\lambda(\boldsymbol{d}, Q))$ instead of $\Phi\left(\lambda(\boldsymbol{d}, Q), Q^{\prime}\right)$.

6.14. Example. Suppose that $2 n=26, p=14, q=13$ and $\boldsymbol{d}=2[4]^{+} \oplus 3[4]^{-} \oplus$ $[3]^{+} \oplus[3]^{-}$. We see that $\left[r_{x_{0}}^{y_{0}}\right]^{*}=[3]^{+}$for any admissible order $Q$ on the set of rows of $\boldsymbol{d}$. Hence, $\left(p^{\prime}, q^{\prime}\right)=\operatorname{sgn}\left([3]^{+} \oplus[3]^{-}\right)=(3,3),\left(p^{\prime}+p-n, q^{\prime}+q-n\right)=(4,3)$, $u=2, v=3$ and $\sigma=\min \{4-3,3-2\}=1$. Now, $r_{x_{0}}^{y_{0}}=3$ is odd, $y_{0}=2$ is even, $\left[r_{x_{0}}^{y_{0}}\right]^{*}$ is a positive row, and $\sigma \neq 0$. So we are in case (iii) of 6.10. Therefore, $\lambda(\boldsymbol{d}, Q)=2[4]^{+} \oplus 3[4]^{-} \oplus 3[1]^{+} \oplus 3[1]^{-}$for all four different admissible orders $Q$. It is obvious that $\lambda(\boldsymbol{d}, Q) \leq \boldsymbol{d}$. Now, $\psi_{1}(\lambda(\boldsymbol{d}, Q))=2[4]^{+} \oplus 3[4]^{-} \oplus 3[1]^{+} \oplus 2[1]^{-}$ and $\psi_{2}(\lambda(\boldsymbol{d}, Q))=[1]^{+} \oplus[1]^{-}$. Therefore, $\Phi(\lambda(\boldsymbol{d}, Q))=3[5]^{+} \oplus 2[5]^{-} \oplus[1]^{+} \oplus[1]^{-}$. It is not difficult to check that there are two signed Young diagrams $\left(\boldsymbol{d}, Q_{1}\right)$ and $\left(\boldsymbol{d}, Q_{2}\right)$ of $\boldsymbol{d}$ such that

$$
\begin{aligned}
& \Phi\left(\boldsymbol{d}, Q_{1}\right)=2[5]^{+} \oplus 2[5]^{-} \oplus[3]^{+} \oplus[2]^{+} \oplus[2]^{-}, \\
& \Phi\left(\boldsymbol{d}, Q_{2}\right)=3[5]^{+} \oplus[5]^{-} \oplus[3]^{-} \oplus[2]^{+} \oplus[2]^{-} .
\end{aligned}
$$

Clearly, $\Phi(\lambda(\boldsymbol{d}, Q))>\Phi\left(\boldsymbol{d}, Q_{1}\right)$ and $\Phi(\lambda(\boldsymbol{d}, Q))>\Phi\left(\boldsymbol{d}, Q_{2}\right)$. 


\section{The orbit correspondences for real reductive dual pairs}

7.1. Keep the assumption that $F:=\mathbb{R}$, and let $\varphi, \Phi$ be the maps defined in 6.6.

Proposition. If $\mathcal{O}$ is a nilpotent orbit in $\mathfrak{s p}(V)$ such that $\mathcal{O}$ is in the image of $\tau_{1}$, then $\Phi(\mathbb{O})$ is the union of maximal elements in the set of nilpotent orbits contained in $\tau_{2}\left(\tau_{1}^{-1}(\mathcal{O})\right)$.

The proof of this proposition is in 7.4.

7.2. Complexification. Let $W^{\mathbb{C}}:=W \otimes_{\mathbb{R}} \mathbb{C}$. The real Lie algebra $\mathfrak{s o}(W)$ is naturally contained in the complex Lie algebra $\mathfrak{s o}\left(W^{\mathbb{C}}\right)$. Hence, a real orbit 2 in $\mathfrak{s o}(W)$ is contained in a unique complex orbit in $\mathfrak{s o}\left(W^{\mathbb{C}}\right)$, denoted by $2^{\mathbb{C}}$. The complex orbit $2^{\mathbb{C}}$ is called the complexification of 2 . If $\boldsymbol{d}$ is the signed partition corresponding to the real orbit 2 , then we can obtain the partition corresponding to the complex orbit $2^{\mathbb{C}}$ by forgetting the signs in $\boldsymbol{d}$.

Lemma. Let $\mathrm{O}_{\boldsymbol{d}}$ be a nilpotent orbit contained in the image of $\tau_{1}$. If $2_{1}$ and $\mathscr{2}_{2}$ are two nilpotent orbits contained in $\Phi\left(\mathbb{O}_{d}\right)$, then $2_{1}^{\mathbb{C}}=2_{2}^{\mathbb{C}}$.

Proof. Let $Q_{1}$ and $Q_{2}$ be the orders of rows of $\psi_{1}(\boldsymbol{d})$ such that $2_{1}$ and $\mathscr{2}_{2}$ are nilpotent orbits corresponding to

$$
\Phi^{*}\left(\psi_{1}(\boldsymbol{d}), Q_{1}\right) \oplus \psi_{2}(\boldsymbol{d}) \quad \text { and } \quad \Phi^{*}\left(\psi_{1}(\boldsymbol{d}), Q_{2}\right) \oplus \psi_{2}(\boldsymbol{d}),
$$

respectively. From the construction, it is clear that the left summands have the same complexification. Hence, the sums have the same complexification.

7.3. Lemma. Let $\mathcal{O}$ be a nilpotent orbit in the image of $\tau_{1}$. Suppose that $\mathscr{P}$ is a nilpotent orbit contained in $\tau_{2}\left(\tau_{1}^{-1}(\mathbb{O})\right)$. There exists a nilpotent orbit 2 contained in $\Phi(\mathbb{O})$ such that $\mathscr{P} \leq 2$.

Proof. Let $V^{\mathbb{C}}:=V \otimes_{\mathbb{R}} \mathbb{C}$ and $W^{\mathbb{C}}:=W \otimes_{\mathbb{R}} \mathbb{C}$. Let $\tau_{1}^{\mathbb{C}}: V^{\mathbb{C}} \otimes_{\mathbb{C}} W^{\mathbb{C}} \rightarrow \mathfrak{s p}\left(V^{\mathbb{C}}\right)$ and $\tau_{2}^{\mathbb{C}}: V^{\mathbb{C}} \otimes_{\mathbb{C}} W^{\mathbb{C}} \rightarrow \mathfrak{s o}\left(W^{\mathbb{C}}\right)$ be the complex moment maps. Clearly, the complex nilpotent orbit $\mathscr{P} \mathbb{C}$ is contained in $\tau_{2}^{\mathbb{C}}\left(\tau_{1}^{\mathbb{C}^{-1}}\left(\mathbb{O}^{\mathbb{C}}\right)\right)$. Moreover, if $\mathscr{2}^{\prime}$ is a nilpotent orbit contained in $\Phi(\mathbb{O})$, then clearly $2^{\prime \mathbb{C}}=\Phi\left(0^{\mathbb{C}}\right)$, where $\Phi\left(0^{\mathbb{C}}\right)$ is as defined in 4.4. Hence, we have $\mathscr{P} \mathbb{C} \leq 2^{\mathbb{C}}$ by 4.6. Clearly, we need only consider the case when there is no nilpotent orbit $\mathscr{P}^{\prime} \subseteq \tau_{2}\left(\tau_{1}^{-1}(\mathbb{O})\right)$ such that $\mathscr{P}<\mathscr{P}^{\prime}$ and $\mathscr{P}^{\prime} \mathbb{C} \leq 2^{\mathbb{C}}$.

Let $\boldsymbol{d}=\bigoplus_{i=1}^{s} \bigoplus_{j=1}^{\gamma_{i}}\left[r_{i}^{j}\right]^{*}$ be the symplectic signed partition corresponding to the nilpotent orbit 0 . From 5.1, 5.2 and 5.3, we know that a nilpotent orbit contained in $\tau_{2}\left(\tau_{1}^{-1}(\mathcal{O})\right)$ should correspond to an orthogonal signed partition $\boldsymbol{e}$, constructed from $\boldsymbol{d}$ according the following rules:

(i) If $\left[r_{i}^{j}\right]^{+}$(respectively, $\left[r_{i}^{j}\right]^{-}$) is an even single row of $\boldsymbol{d}$, then $\boldsymbol{e}$ has an odd single row $\left[r_{i}^{j}+1\right]^{-}$or $\left[r_{i}^{j}+1\right]^{-}$(respectively, $\left[r_{i}^{j}+1\right]^{+}$or $\left[r_{i}^{j}+1\right]^{+}$); that is, we can remove or add a signed box to an even single row of $\boldsymbol{d}$. We shall say that the row $\left[r_{i}^{j}+1\right]^{*}$ or $\left[r_{i}^{j}-1\right]^{*}$ is the image in $\boldsymbol{e}$ of the row $\left[r_{i}^{j}\right]^{*}$. 
(ii) If $\left[r_{i}^{j-1}\right]^{+} \oplus\left[r_{i}^{j}\right]^{-}$is an odd double row, then $\boldsymbol{e}$ has a double row

$$
\left[r_{i}^{j-1}-1\right]^{-} \oplus\left[r_{i}^{j}-1\right]^{+}, \quad\left[r_{i}^{j-1}\right]^{+} \oplus\left[r_{i}^{j}\right]^{-}, \quad \text { or } \quad\left[r_{i}^{j-1}+1\right]^{-} \oplus\left[r_{i}^{j}+1\right]^{+} .
$$

(iii) The signature of $\boldsymbol{e}$ is $(p, q)$.

Suppose that $\mathscr{P} \not \mathscr{Q}^{\prime}$, for otherwise the lemma is proved. Give an order on the set of rows of $\boldsymbol{d}$ as in Equation (6-3). Let $\left[r_{x_{1}}^{y_{1}}\right]^{*}$ be the largest row in $\boldsymbol{d}$ whose image in the signed partition corresponding to $\mathscr{P}$ has longer length than its image in the signed partition corresponding to $2^{\prime}$. From the construction of $\Phi(0)$, we see that $\boldsymbol{d}$ has a row $\left[r_{x_{2}}^{y_{2}}\right]^{*}$ such that its image in the signed partition corresponding to $2^{\prime}$ has longer length than that in the signed partition corresponding to $\mathscr{P}$, and $r_{x_{2}}^{y_{2}} \geq r_{x_{1}}^{y_{1}}$. However, $\mathscr{P} \not \leq \mathscr{Q}^{\prime}$, and there is no nilpotent orbit $\mathscr{P}^{\prime}$ contained in $\tau_{2}\left(\tau_{1}^{-1}(\mathcal{O})\right)$ such that $\mathscr{P}<\mathscr{P}^{\prime}$ and $\mathscr{P}^{\prime \mathbb{C}} \leq \mathscr{Q}^{\mathbb{C}}$, so $r_{x_{2}}^{y_{2}}$ is equal to $r_{x_{1}}^{y_{1}}$, while $\left[r_{x_{1}}^{y_{1}}\right]^{*}$ and $\left[r_{x_{2}}^{y_{2}}\right]^{*}$ have opposite parity. Then, there is a nilpotent orbit $2^{\prime \prime} \in \Phi(\mathbb{O})$ such that the largest row in $\boldsymbol{d}$, with its image in the signed partition corresponding to $\mathscr{P}$ having longer length than its image in the signed partition corresponding to $2^{\prime \prime}$, is strictly smaller than $\left[r_{x_{1}}^{y_{1}}\right]^{*}$. Hence, the result can be obtained by induction.

7.4. Proof of the proposition in 7.1. It is clear from the construction and the rules in 5.1, 5.2 and 5.3 that every nilpotent 2 in $\Phi(0)$ is contained in $\tau_{2}\left(\tau_{1}^{-1}(\mathbb{O})\right)$. Suppose that $2_{1}$ and $\mathscr{2}_{2}$ are two different nilpotent orbits contained in $\Phi(0)$. From the construction, we know that $\mathscr{2}_{1}$ and $\mathscr{2}_{2}$ have the same complexification. Therefore, they are clearly not comparable. Suppose that $\mathscr{P}$ is a nilpotent orbit contained in $\tau_{2}\left(\tau_{1}^{-1}(\mathcal{O})\right)$. Then, by $7.3, \mathscr{P} \leq 2$ for some nilpotent orbit 2 contained in $\Phi(\mathbb{O})$. Hence, every nilpotent orbit contained in $\Phi(\mathcal{O})$ is a maximal element in the ordered set of nilpotent orbits contained in $\tau_{2}\left(\tau_{1}^{-1}(\mathcal{O})\right)$. Moreover, every maximal element in the set of nilpotent orbits contained in $\tau_{2}\left(\tau_{1}^{-1}(\mathcal{O})\right)$ is contained in $\Phi(\mathcal{O})$.

7.5. Proposition. If $\mathrm{O}_{1}$ and $\mathrm{O}_{2}$ are nilpotent orbits in $\mathfrak{s p}(V)$ such that $\mathrm{O}_{1} \geq \mathrm{O}_{2}$, then $\varphi\left(\mathrm{O}_{1}\right) \geq \varphi\left(\mathrm{O}_{2}\right)$. Moreover, $\varphi\left(\mathrm{O}_{)}\right.$is the unique minimal element among the nilpotent orbits contained in $\tau_{2}\left(\tau_{1}^{-1}(0)\right)$.

Proof. Use the definition of $\varphi$ in 6.6 and the rules in 5.1, 5.2 and 5.3.

7.6. Lemma. If $\mathfrak{O}_{\boldsymbol{d}}$ is a nilpotent orbit contained in the image of $\tau_{1}$, then $\mathscr{O}_{\Phi(\lambda(\boldsymbol{d}, Q))}$ is a maximal element in the set of nilpotent orbits in $\tau_{2}\left(\tau_{1}^{-1}\left(\overline{\mathbb{O}}_{\boldsymbol{d}}\right)\right)$. Every maximal element in the set of nilpotent orbits in $\tau_{2}\left(\tau_{1}^{-1}\left(\overline{\mathbb{O}}_{d}\right)\right)$ is of this form for some $Q$.

Proof. Suppose that $\mathscr{O}_{\Phi(\lambda(d, Q))}$ is not a maximal element. Then, $\mathbb{O}_{\Phi(\lambda(d, Q))}<\mathscr{P}$ for some nilpotent orbit $\mathscr{P}$ in $\tau_{2}\left(\tau_{1}^{-1}\left(\overline{0}_{\boldsymbol{d}}\right)\right)$. Hence, $O_{\Phi(\lambda(\boldsymbol{d}, Q))}^{\mathbb{C}}<\mathscr{P} \mathbb{C}$, contradicting 4.9. It is also clear from 4.9 that every maximal element in the set of nilpotent orbits in $\tau_{2}\left(\tau_{1}^{-1}\left(\overline{\mathscr{O}}_{\boldsymbol{d}}\right)\right)$ is of the form $\mathbb{O}_{\Phi(\lambda(\boldsymbol{d}, Q))}$ for some $Q$. 
7.7. Lemma. If $\mathscr{O}_{\boldsymbol{d}_{1}}, \mathrm{O}_{\boldsymbol{d}_{2}}$ are two nilpotent orbits contained in the image of $\tau_{1}$ such that $\mathcal{O}_{\boldsymbol{d}_{1}} \leq \mathrm{O}_{\boldsymbol{d}_{2}}$, then, for every nilpotent orbit $2_{1}$ contained in $\bigcup_{\left(\boldsymbol{d}_{1}, Q_{1}\right)} \mathcal{O}_{\Phi\left(\lambda\left(\boldsymbol{d}_{1}, Q_{1}\right)\right)}$, there is a nilpotent orbit $2_{2}$ contained in $\bigcup_{\left(d_{2}, Q_{2}\right)} \mathcal{O}_{\Phi\left(\lambda\left(d_{2}, Q_{2}\right)\right)}$ such that $\mathscr{2}_{1} \leq 2_{2}$, where the union $\bigcup_{\left(d_{i}, Q_{i}\right)}$ runs over the admissible signed Young diagrams of the signed partition $\boldsymbol{d}_{i}$.

Proof. Suppose $\left(\boldsymbol{d}_{1}, Q_{1}\right)$ is an admissible signed Young diagram of $\boldsymbol{d}_{1}$ such that $2_{1}=\mathscr{O}_{\Phi\left(\lambda\left(\boldsymbol{d}_{1}, Q_{1}\right)\right)}$. We have $\lambda\left(\boldsymbol{d}_{1}, Q_{1}\right) \leq \boldsymbol{d}_{1} \leq \boldsymbol{d}_{2}$. So, $\mathscr{O}_{\Phi\left(\lambda\left(\boldsymbol{d}_{1}, Q_{1}\right)\right)} \subset \tau_{2}\left(\tau_{1}^{-1}\left(\overline{\mathcal{O}}_{\boldsymbol{d}_{2}}\right)\right)$. By 6.12, we know that a maximal element in the set of nilpotent orbits contained in $\tau_{2}\left(\tau_{1}^{-1}\left(\overline{\mathcal{O}}_{\boldsymbol{d}_{2}}\right)\right)$ is equal to $\mathrm{O}_{\Phi\left(\lambda\left(\boldsymbol{d}_{2}, Q_{2}\right)\right)}$ for some admissible signed Young diagram $\left(\boldsymbol{d}_{2}, Q_{2}\right)$ of $\boldsymbol{d}_{2}$. Hence, $2_{1} \leq \mathcal{O}_{\Phi\left(\lambda\left(\boldsymbol{d}_{2}, Q_{2}\right)\right)}$.

7.8. Proof of 6.1. First, we show that

$$
\tau_{2}\left(\tau_{1}^{-1}\left(\overline{\mathscr{O}}_{\boldsymbol{d}}\right)\right) \subseteq \bigcup_{(\boldsymbol{d}, Q)} \overline{\bar{O}_{\Phi(\lambda(\boldsymbol{d}, Q))}} .
$$

Suppose 2 is a nilpotent orbit in $\tau_{2}\left(\tau_{1}^{-1}\left(\overline{\mathscr{O}}_{\boldsymbol{d}}\right)\right)$. Then, 2 is in $\tau_{2}\left(\tau_{1}^{-1}\left(\mathscr{O}_{\boldsymbol{d}_{1}}\right)\right)$ for some nilpotent orbit $\mathscr{O}_{\boldsymbol{d}_{1}}$ in $\mathfrak{s p}(V)$ such that $\mathscr{O}_{\boldsymbol{d}_{1}} \leq \mathrm{O}_{\boldsymbol{d}}$. From 7.1, we know that $2 \leq 2_{1}$ for some nilpotent orbit $2_{1}$ contained in $\Phi\left(\mathcal{O}_{d_{1}}\right)$. By 7.6, there exists an admissible signed Young diagram $\left(\boldsymbol{d}_{1}, Q_{1}\right)$ such that $2_{1} \leq \mathrm{O}_{\Phi\left(\lambda\left(\boldsymbol{d}_{1}, Q_{1}\right)\right)}$. Since $\boldsymbol{O}_{\boldsymbol{d}_{1}} \leq \mathrm{O}_{\boldsymbol{d}}$, by 7.7, there is an admissible signed Young diagram $(\boldsymbol{d}, Q)$ such that $\mathbb{O}_{\Phi\left(\lambda\left(\boldsymbol{d}_{1}, Q_{1}\right)\right) \leq} \leq$ $\mathcal{O}_{\Phi(\lambda(\boldsymbol{d}, Q))}$. Hence, $2_{1} \leq \mathcal{O}_{\Phi(\lambda(\boldsymbol{d}, Q))}$ and therefore $\mathscr{2}_{1} \subseteq \bigcup_{(\boldsymbol{d}, Q)} \overline{\mathrm{O}_{\Phi(\lambda(\boldsymbol{d}, Q))}}$.

Next, we prove the opposite inclusion

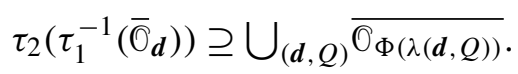

Let $\mathscr{2}^{\prime}$ be a nilpotent orbit contained in $\bigcup_{(\boldsymbol{d}, Q)} \overline{\mathrm{O}_{\Phi(\lambda(\boldsymbol{d}, Q))}}$. Then, $\mathscr{2}^{\prime} \leq \mathrm{O}_{\Phi(\lambda(\boldsymbol{d}, Q))}$ for some admissible signed Young diagram $(\boldsymbol{d}, Q)$. By a construction similar to 6.6, we can define a map $\varphi^{\prime}$, from the set of nilpotent orbits in $\mathfrak{s o}(W)$ to the set of nilpotent orbits in $\mathfrak{s p}(V)$, such that $\varphi^{\prime}\left(\mathcal{O}^{\prime}\right)$ is the unique minimal element among the nilpotent orbits contained in $\tau_{1}\left(\tau_{2}^{-1}\left({\mathcal{O}^{\prime}}^{\prime}\right)\right.$ ) for any nilpotent orbit ${O^{\prime}}^{\prime}$ in $\mathfrak{s o}(W)$. Moreover,

$$
\varphi^{\prime}\left(2^{\prime}\right) \leq \varphi^{\prime}\left(\mathcal{O}_{\Phi(\lambda(\boldsymbol{d}, Q))}\right) .
$$

We know that $\mathscr{O}_{\Phi(\lambda(\boldsymbol{d}, Q))}$ is contained in $\tau_{2}\left(\tau_{1}^{-1}\left(\mathscr{O}_{\lambda(\boldsymbol{d}, Q)}\right)\right)$. Hence, the nilpotent orbit $\mathbb{O}_{\lambda(\boldsymbol{d}, Q)}$ is contained in $\tau_{1}\left(\tau_{2}^{-1}\left(\mathbb{O}_{\Phi(\lambda(\boldsymbol{d}, Q))}\right)\right)$. Thus, $\varphi^{\prime}\left(\mathbb{O}_{\Phi(\lambda(\boldsymbol{d}, Q))}\right) \leq \mathbb{O}_{\lambda(\boldsymbol{d}, Q)}$ because $\varphi^{\prime}\left(\mathscr{O}_{\Phi(\lambda(\boldsymbol{d}, Q))}\right)$ is the unique minimal element among the nilpotent orbits contained in $\tau_{1}\left(\tau_{2}^{-1}\left(\mathcal{O}_{\Phi(\lambda(\boldsymbol{d}, Q))}\right)\right)$. Hence, we have $\varphi^{\prime}\left(2^{\prime}\right) \leq \mathcal{O}_{\lambda(\boldsymbol{d}, Q)}$ from (7-1). Then, by 6.13 , we have

$$
\varphi^{\prime}\left(2^{\prime}\right) \leq \mathrm{O}_{\boldsymbol{d}} .
$$

From the construction, we know that $\varphi^{\prime}\left(\mathscr{Q}^{\prime}\right)$ is contained in $\tau_{1}\left(\tau_{2}^{-1}\left(\mathscr{Q}^{\prime}\right)\right)$. Hence, $\mathscr{2}^{\prime}$ is contained in $\tau_{2}\left(\tau_{1}^{-1}\left(\varphi^{\prime}\left(2^{\prime}\right)\right)\right.$. By $(7-2)$, we see that $2^{\prime}$ is contained in $\tau_{2}\left(\tau_{1}^{-1}\left(\overline{\mathbb{O}}_{\boldsymbol{d}}\right)\right)$. 
From 6.12, we know that $\bigcup_{(d, Q)} \overline{\sigma_{\Phi(\lambda(d, Q))}}$ is a union of at most two closures of nilpotent orbits.

\section{Acknowledgments}

The first draft of this paper was finished in the summer of 1995. I like to thank Professor Barbasch deeply for suggesting this subject and teaching me the basic material. In fact most parts of sections 3 and 5 are rewritten from his note. I also thank the referee for numerous useful suggestions.

\section{References}

[Adams 1983] J. D. Adams, "Discrete spectrum of the reductive dual pair $(\mathrm{O}(p, q), \mathrm{Sp}(2 m))$ ", Invent. Math. 74:3 (1983), 449-475. MR 85k:22031 Zbl 0561.22011

[Adams 1987] J. Adams, "Coadjoint orbits and reductive dual pairs", Adv. in Math. 63:2 (1987), 138-151. MR 88c:22012 Zbl 0623.22015

[Collingwood and McGovern 1993] D. H. Collingwood and W. M. McGovern, Nilpotent orbits in semisimple Lie algebras, Van Nostrand Reinhold, New York, 1993. MR 94j:17001 Zbl 0972.17008

[Daszkiewicz et al. 1997] A. Daszkiewicz, W. Kraśkiewicz, and T. Przebinda, "Nilpotent orbits and complex dual pairs”, J. Algebra 190:2 (1997), 518-539. MR 98b:20063 Zbl 0870.22007

[Djoković 1981] D. Ž. Djoković, "Closures of conjugacy classes in classical real linear Lie groups", pp. 63-83 in Algebra (Carbondale, IL, 1980), edited by R. K. Amayo, Lecture Notes in Math. 848, Springer, Berlin, 1981. MR 82h:20052 Zbl 0462.22003

[He 2003] H. He, “Unipotent representations and quantum induction”, preprint, 2003. arXiv math. RT/0210372

[He 2005] H. He, "Compositions of theta correspondences", Adv. Math. 190:2 (2005), 225-263. MR 2005h:22021 Zbl 1059.22014

[Howe 1989] R. Howe, "Remarks on classical invariant theory", Trans. Amer. Math. Soc. 313:2 (1989), 539-570. MR 90h:22015a Zbl 0674.15021

[Joseph 1985] A. Joseph, "On the associated variety of a primitive ideal", J. Algebra 93:2 (1985), 509-523. MR 86m:17014 Zbl 0594.17009

[Kazhdan et al. 1978] D. Kazhdan, B. Kostant, and S. Sternberg, "Hamiltonian group actions and dynamical systems of Calogero type", Comm. Pure Appl. Math. 31:4 (1978), 481-507. MR 57 \#17711 Zbl 0368.58008

[Przebinda 1993] T. Przebinda, "Characters, dual pairs, and unitary representations", Duke Math. J. 69:3 (1993), 547-592. MR 94i:22036 Zbl 0788.22018

Received June 29, 2008. Revised September 15, 2010.

SHU-YEN PAN

NATIONAL TSING HUA UNIVERSITY

DEPARTMENT OF MATHEMATICS

No. 101, SECTION 2, KUANG-Fu RoAD

HSINCHU 300

TAIWAN

sypan@math.nthu.edu.tw 


\title{
PACIFIC JOURNAL OF MATHEMATICS
}

\author{
http://www.pjmath.org \\ Founded in 1951 by \\ E. F. Beckenbach (1906-1982) and F. Wolf (1904-1989)
}

\section{EDITORS}

V. S. Varadarajan (Managing Editor)

Department of Mathematics

University of California

Los Angeles, CA 90095-1555

pacific@math.ucla.edu

Vyjayanthi Chari

Department of Mathematics

University of California

Riverside, CA 92521-0135

chari@math.ucr.edu

Robert Finn

Department of Mathematics Stanford University

Stanford, CA 94305-2125

finn@math.stanford.edu

Kefeng Liu

Department of Mathematics

University of California

Los Angeles, CA 90095-1555

liu@math.ucla.edu
Darren Long

Department of Mathematics

University of California

Santa Barbara, CA 93106-3080

long@math.ucsb.edu

Jiang-Hua Lu

Department of Mathematics

The University of Hong Kong

Pokfulam Rd., Hong Kong jhlu@maths.hku.hk

Alexander Merkurjev

Department of Mathematics

University of California

Los Angeles, CA 90095-1555

merkurev@math.ucla.edu
Sorin Popa

Department of Mathematics University of California

Los Angeles, CA 90095-1555 popa@math.ucla.edu

Jie Qing

Department of Mathematics

University of California

Santa Cruz, CA 95064

qing@cats.ucsc.edu

Jonathan Rogawski

Department of Mathematics

University of California

Los Angeles, CA 90095-1555

jonr@math.ucla.edu

\section{PRODUCTION}

pacific@math.berkeley.edu

\begin{abstract}
Silvio Levy, Scientific Editor Matthew Cargo, Senior Production Editor
\end{abstract}
ACADEMIA SINICA, TAIPEI

CALIFORNIA INST. OF TECHNOLOGY

INST. DE MATEMÁTICA PURA E APLICADA

KEIO UNIVERSITY

MATH. SCIENCES RESEARCH INSTITUTE

NEW MEXICO STATE UNIV.

OREGON STATE UNIV.

\section{SUPPORTING INSTITUTIONS}

STANFORD UNIVERSITY
UNIV. OF BRITISH COLUMBIA
UNIV. OF CALIFORNIA, BERKELEY
UNIV. OF CALIFORNIA, DAVIS
UNIV. OF CALIFORNIA, LOS ANGELES
UNIV. OF CALIFORNIA, RIVERSIDE
UNIV. OF CALIFORNIA, SAN DIEGO
UNIV. OF CALIF., SANTA BARBARA

UNIV. OF CALIF., SANTA CRUZ

UNIV. OF MONTANA

UNIV. OF OREGON

UNIV. OF SOUTHERN CALIFORNIA

UNIV. OF UTAH

UNIV. OF WASHINGTON

WASHINGTON STATE UNIVERSITY

These supporting institutions contribute to the cost of publication of this Journal, but they are not owners or publishers and have no responsibility for its contents or policies.

See inside back cover or www.pjmath.org for submission instructions.

The subscription price for 2010 is US \$420/year for the electronic version, and \$485/year for print and electronic.

Subscriptions, requests for back issues from the last three years and changes of subscribers address should be sent to Pacific Journal of Mathematics, P.O. Box 4163, Berkeley, CA 94704-0163, U.S.A. Prior back issues are obtainable from Periodicals Service Company, 11 Main Street, Germantown, NY 12526-5635. The Pacific Journal of Mathematics is indexed by Mathematical Reviews, Zentralblatt MATH, PASCAL CNRS Index, Referativnyi Zhurnal, Current Mathematical Publications and the Science Citation Index.

The Pacific Journal of Mathematics (ISSN 0030-8730) at the University of California, c/o Department of Mathematics, 969 Evans Hall, Berkeley, CA 94720-3840, is published monthly except July and August. Periodical rate postage paid at Berkeley, CA 94704, and additional mailing offices. POSTMASTER: send address changes to Pacific Journal of Mathematics, P.O. Box 4163, Berkeley, CA 94704-0163.

PJM peer review and production are managed by EditFLOW ${ }^{\mathrm{TM}}$ from Mathematical Sciences Publishers.

PUBLISHED BY PACIFIC JOURNAL OF MATHEMATICS

at the University of California, Berkeley 94720-3840

A NON-PROFIT CORPORATION

Typeset in LATEX

Copyright $(\mathrm{C} 2010$ by Pacific Journal of Mathematics 


\section{PACIFIC JOURNAL OF MATHEMATICS}

Volume $248 \quad$ No. $2 \quad$ December 2010

Topological description of Riemannian foliations with dense leaves

Jesús A. ÁlVAREZ LóPEZ and Alberto CANDEL

The nonexistence of quasi-Einstein metrics

JEFFREY S. CASE

Twisted symmetric group actions

AKINARI HoSHI and MING-CHANG KANG

Optimal transportation and monotonic quantities on evolving manifolds

305

HONG HUANG

Hopf structures on the Hopf quiver $Q(\langle g\rangle, g)$

HuA-Lin HuANG, YU Ye and QING ZHaO

Minimal surfaces in $S^{3}$ foliated by circles

Nikolai KuteV and VelichKa Milousheva

Prealternative algebras and prealternative bialgebras

XIANG Ni and CHENGMING BAI

Some remarks about closed convex curves

Ke OU and Shengliang PAN

Orbit correspondences for real reductive dual pairs

SHU-YEN PAN

Graphs of bounded degree and the $p$-harmonic boundary

Michael J. Puls

Invariance of the BFV complex

FLORIAN SCHÄTZ

Some elliptic PDEs on Riemannian manifolds with boundary

YANNICK SIRE and ENRICO VALDINOCI

Representations of Lie superalgebras in prime characteristic, III 